\title{
WestVirginiaUniversity
}

THE RESEARCH REPOSITORY @ WVU

Graduate Theses, Dissertations, and Problem Reports

2000

\section{Symmetrically continuous functions}

\author{
Marcin Szyszkowski \\ West Virginia University
}

Follow this and additional works at: https://researchrepository.wvu.edu/etd

\section{Recommended Citation}

Szyszkowski, Marcin, "Symmetrically continuous functions" (2000). Graduate Theses, Dissertations, and Problem Reports. 3186.

https://researchrepository.wvu.edu/etd/3186

This Dissertation is protected by copyright and/or related rights. It has been brought to you by the The Research Repository @ WVU with permission from the rights-holder(s). You are free to use this Dissertation in any way that is permitted by the copyright and related rights legislation that applies to your use. For other uses you must obtain permission from the rights-holder(s) directly, unless additional rights are indicated by a Creative Commons license in the record and/ or on the work itself. This Dissertation has been accepted for inclusion in WVU Graduate Theses, Dissertations, and Problem Reports collection by an authorized administrator of The Research Repository @ WVU.

For more information, please contact researchrepository@mail.wvu.edu. 
Symmetrically Continuous Functions

\author{
Marcin Szyszkowski \\ Dissertation submitted to the \\ College of Arts and Sciences \\ at West Virginia University \\ The degree of \\ Doctor of Philosophy \\ in \\ Mathematics
} In partial fulfillment of the Requirements for

Chris K. Ciesielski, Ph.D., Chair Harvey Diamond, Ph.D. John Littleton, Ph.D. Sam Nadler, Ph.D. Jerzy Wojciechowski, Ph.D. Janusz Pawlikowski, Ph.D. Depatrment of Mathematics Morgantown, West Virginia 2000

Keywords: Continuity, Symmetric Continuity, Measurability 


\section{ABSTRACT \\ Symmetrically Continuous Functions}

\section{Marcin Szyszkowski}

This work is devoted entirely to symmetric continuity and consists of five chapters. Chapter 1 is an introduction. Chapter 2, based on the paper [Sz2], is the main part of this work. In this chapter we generalize classic theorems on functions defined on the intervals to functions defined on arbitrary subsets of the real line. Chapter 3 deals with a weaker condition then symmetric continuity — weak symmetric continuity. It is an extended version of my paper [Sz1]. Chapter 4 is based on the paper [CSz]. Here it is shown that symmetrically continuous functions, although being "close" to continuous functions, may not be nice in some sense. The last chapter is about the symmetric derivative and relates back to the Chapter 2. 


\section{Contents}

1 Introduction 1

$1.1 \ldots \ldots \ldots \ldots \ldots \ldots$

2 Functions on subsets of R 3

2.1 Chapter overview .................... . . . 3

2.2 Continuity of symmetrically continuous functions . . . . . . . . . 7

2.3 Proof of the main result . . . . . . . . . . . . . . . . . 11

2.4 Extensions of continuous and symmetrically continuous functions . . 21

3 Weak symmetric continuity 26

$3.1 \ldots \ldots \ldots \ldots \ldots \ldots \ldots$

4 "Bad" symmetrically continuous functions $\quad 36$

4.1 Introduction . . . . . . . . . . . . . . . . 36

4.2 Technical Lemmas . . . . . . . . . . . . . . . . . . . . . . . . . . . . . . . . . . . . . 47

4.3 Main result . . . . . . . . . . . . . . . . . . . . . . 40

4.4 Continuity cardinal . . . . . . . . . . . . . . . . . . 42

5 Symmetric derivative $\quad 46$

5.1 Symmetric and ordinary derivatives . . . . . . . . . . . . . 46

5.2 Measurability of symmetric derivatives . . . . . . . . . . . 51 


\section{Chapter 1}

\section{Introduction}

\section{1}

The theory of symmetric and symmetrically continuous functions dates back to the beginning of twentieth century. First publications about symmetrically continuous functions appeared in the 1910's. Since then, in every decade we have seen some papers about this topic. Recently, in the 1980's and early 1990's we have witnessed a particular interest in symmetric continuity. The theory stems from the theory of trigonometric series and, as is usually the case, evolved into a separate branch of mathematics. It never, however, broke its ties to trigonometric series. People call the theory symmetric analysis after Lee Larson [L].

All functions in our work are defined on subsets of $\mathbf{R}$, the real line, and have values in $\mathbf{R}$. The central notion is defined as follows.

Definition 1.1.1 A function $f: \mathbf{R} \rightarrow \mathbf{R}$ is symmetrically continuous at a point $x$ if

$$
\forall_{\varepsilon>0} \exists_{\delta>0} \forall_{|h|<\delta} \quad|f(x+h)-f(x-h)|<\varepsilon
$$


or, equivalently,

$$
\lim _{h \rightarrow 0}[f(x+h)-f(x-h)]=0
$$

If the function is not symmetrically continuous at a point, we will say it is symmetrically discontinuous there.

A congenial notion is symmetry which says that

$$
\lim _{h \rightarrow 0}[f(x+h)+f(x-h)-2 f(x)]=0 .
$$

Both properties have been deeply studied and very often there is an analogy between them. However, we will confine ourselves only to the first notion.

Obviously ordinary continuity implies symmetric continuity, but not the other way around. Let us give some simple examples. The discontinuous function $f(x)=0$ for $x \neq 0$ and $f(0)=1$ is symmetrically continuous everywhere. The function $\cos \left(\frac{1}{x}\right)$ (where we put any value at $x=0$ ) is symmetrically continuous everywhere (including 0 ) but $\sin \left(\frac{1}{x}\right)$ is not symmetrically continuous at 0 .

We will often utilize variations of the following example.

Example 1.1.2 Let $G \subset \mathbf{R}$ be an additive group and $f=\chi_{G}: \mathbf{R} \rightarrow\{0,1\}$ be the characteristic function of $G$. Then $f$ is symmetrically continuous on $G$.

Indeed if $a \in G$ then for any point $s=a+h$ its symmetric reflection about $a$ is $a-h=2 a-s$ and it belongs to $G$ if and only if $s$ does. Outside $G, f$ is symmetrically continuous only at points $a$ for which $2 a \in G$. In particular if $G$ is divisible by 2 then $f$ is symmetrically discontinuous everywhere outside $G$. 


\section{Chapter 2}

\section{Functions on subsets of $\mathbf{R}$}

\subsection{Chapter overview}

This chapter is based on my paper [Sz2] and is divided into four sections. In Section 2.2 we state the main results about continuity of symmetrically continuous functions. Section 2.3 consists only of the proofs of theorems from Section 2.2. In Section 2.4 we investigate the extension properties of symmetrically continuous functions to bigger domains.

Our notation is standard and follows [Ci2] and [T2]. By an interval we always mean a nontrivial interval (i.e., containing more than one point). For different $a, b \in$ $\mathbf{R}$ we write $[a, b]$ for the interval $[\min (a, b), \max (a, b)]$ (i.e., $[1,2]=[2,1])$.

As said in the introduction the study of symmetric and symmetrically continuous functions stems from the theory of trigonometric series. In the theory of trigonometric series we study functions defined on various subsets of $\mathbf{R}$ or $\mathbf{R}^{n}$, while symmetrically continuous functions have been studied so far only on the whole real line or on intervals. In this chapter we examine basic properties of symmetrically continuous functions defined on arbitrary subsets of $\mathbf{R}$. In particular we will compare these 
properties with those possessed by symmetrically continuous functions defined on the whole real line. Doing so we generalize Theorems 2.2.1 and 2.2.3.

We define symmetrical continuity for a function with a domain being an arbitrary subset of the reals in the following way.

Definition 2.1.1 Let $A \subset \mathbf{R}$. A function $f: A \rightarrow \mathbf{R}$ is said to be symmetrically continuous at a point $x$ if

$$
\forall_{\varepsilon>0} \exists_{\delta>0} \forall_{|h|<\delta} \quad x \pm h \in A \Rightarrow|f(x+h)-f(x-h)|<\varepsilon .
$$

If $f$ is symmetrically continuous at every point of $A$ then we say that $f$ is symmetrically continuous on $A$ or simply symmetrically continuous.

Because of the implication above it is possible (and in fact we will often consider such situation) that $f$ is symmetrically continuous at $x$ only because for every $x+h \in$ $A$ the number $x-h$ is not in $A$. If this happens then we say that $f$ is vacuously symmetrically continuous at this point. Requiring additionally in Definition 2.1.1 that the function is defined on a symmetric domain would not improve our results, as Examples 2.2.10 and 2.2.11 show. Thus, considering only symmetric domains would only narrow our main results, since Theorem 2.2.7 as well as Corollaries 2.2.8 and 2.2.9 are obtained without restrictions of symmetry on domains.

To illustrate the new notion let us give a simple example.

Example 2.1.2 Let $G_{1}$ and $G_{2}$ be additive subgroups of $\mathbf{R}$ with $G_{1} \cap G_{2}=\{0\}$ and let $f=\chi_{G_{1}}: G_{1} \cup G_{2} \rightarrow\{0,1\}$. Then $f$ is symmetrically continuous on its domain.

Just like in Example 1.1.2 for any $a \in G_{i}, i=1,2$, and $s \in \mathbf{R}$, we have $s \in G_{i}$ if and only if $2 a-s \in G_{i}$. So $f$ is symmetrically continuous at $a$. Let us note the contrast 
to Example 1.1.2, where the function $f$ was symmetrically discontinuous in points outside $f^{-1}(1)$.

Note that we do not need function $f$ to be defined at a point $x$ to talk about symmetric continuity at $x$. However we will concentrate only on the points from the domain (except in Theorem 2.1.3).

Using functions defined on domains smaller than $\mathbf{R}$ we may address an old problem of Marcus to characterize the set of points at which a function $f: \mathbf{R} \rightarrow \mathbf{R}$ may be symmetrically continuous. Without solving this problem we state the following theorem.

Theorem 2.1.3 For any set $A \subset \mathbf{R}$ there is a set $X \subset \mathbf{R}$ and a function $f: X \rightarrow$ $\{0,1\}$ that is symmetrically continuous (vacuously) at $x \in \mathbf{R}$ (not necessarily in $X$ ) if and only if $x \in A$.

This theorem follows immediately from Lemma 3.1.7. (Let $X$ be as in Lemma 3.1.7 for the set $\mathbf{R} \backslash A$ and $f: X \rightarrow \mathbf{R}$ be the characteristic function of $\bigcup_{a \notin A}\left\{a+h_{n}^{a}: n<\omega\right\}$. Then $f$ is symmetrically continuous (vacuously) at $x \in \mathbf{R}$ if and only if $x \in A$.)

We summarize the properties of symmetrically continuous functions defined on subsets of $\mathbf{R}$ in the chart below. We assume here that the function $f: A \rightarrow \mathbf{R}$ is symmetrically continuous. The property " $f$ is extendable" means that $f$ has a symmetrically continuous extension, and " $f$ is almost extendable" means that there is a symmetrically continuous function $F$ defined on a set containing $A$ such that $\{x \in A: f(x) \neq F(x)\}$ has measure zero. The property " $f$ can be extended" indicates that there is an extension of $f$ which remains symmetrically continuous at every point of $A$. 
The symbol "+" means that the function $f$ has the property in the left column, "-" means that it does not. Marks "(i..)" refer to short explanations below the table. We also included references to appropriate examples and theorems for fast reference.

\begin{tabular}{|c|c|c|c|c|}
\hline Domain $A$ is $: \rightarrow$ & $\mathbf{R}$ & any set & $\begin{array}{c}\text { measurable or } \\
\text { with the Baire } \\
\text { property }\end{array}$ & symmetric \\
\hline$\downarrow f$ is : $\downarrow$ & & & & \\
\hline $\begin{array}{l}\text { measurable or has } \\
\text { the Baire property }\end{array}$ & $\begin{array}{c}\text { Thm. } 2.2 .2 \\
+\end{array}$ & $\begin{array}{l}\text { (i) } \\
-\end{array}$ & $\begin{array}{c}\text { Cor. } 2.2 .8 \& 2.2 .9 \\
+\end{array}$ & $\begin{array}{c}\text { (i), Ex. } 2.2 .11 \\
-\end{array}$ \\
\hline $\begin{array}{c}\text { continuous almost } \\
\text { everywhere on } A\end{array}$ & $\begin{array}{c}\text { Cor. } 2.2 .6 \\
+\end{array}$ & $\begin{array}{cl}\text { Ex. } & 2.2 .11 \\
& -\end{array}$ & $\begin{array}{c}\text { Cor. } 2.2 .8 \& 2.2 .9 \\
+\end{array}$ & $\begin{array}{cl}\text { Ex. } & 2.2 .11 \\
-\end{array}$ \\
\hline extendable to $\mathbf{R}$ & N/A & $\begin{array}{l}\text { (ii) } \\
-\end{array}$ & $\begin{array}{l}\text { (ii) } \\
-\end{array}$ & $\begin{array}{c}\text { Ex. } \\
2.2 .11\end{array}$ \\
\hline $\begin{array}{l}\text { extendable to a } \\
\text { measurable set or } \\
\text { a set with the } \\
\text { Baire property }\end{array}$ & N/A & Ex. 2.2.11 & $\mathrm{N} / \mathrm{A}$ & Ex. 2.2.11 \\
\hline $\begin{array}{c}\text { almost extendable } \\
\text { to } \mathbf{R}\end{array}$ & $\mathrm{N} / \mathrm{A}$ & $\begin{array}{l}\text { (ii) } \\
-\end{array}$ & $\begin{array}{l}\text { (ii) } \\
-\end{array}$ & $\begin{array}{c}\text { Ex. } 2.2 .11 \\
-\end{array}$ \\
\hline $\begin{array}{l}\text { almost extendable } \\
\text { to a residual set }\end{array}$ & $\mathrm{N} / \mathrm{A}$ & $\begin{array}{l}\text { (iii) } \\
-\end{array}$ & $\begin{array}{c}\text { Cor. } 2.2 .8 \& 2.2 .9 \\
+\end{array}$ & $\begin{array}{c}\text { Ex. } 2.2 .11 \\
-\end{array}$ \\
\hline \multicolumn{5}{|c|}{ extensions preserving only original points of symmetric continuity } \\
\hline $\begin{array}{c}\text { can be extended } \\
\text { to } \mathbf{R}\end{array}$ & $\mathrm{N} / \mathrm{A}$ & $\begin{array}{c}\text { Thm. } 2.4 .2 \\
-\end{array}$ & $\begin{array}{c}\text { Ex. } 2.1 .2 \\
-\end{array}$ & $\begin{array}{l}\text { (iv) } \\
+\end{array}$ \\
\hline $\begin{array}{l}\text { can be extended } \\
\text { to a residual set }\end{array}$ & $\mathrm{N} / \mathrm{A}$ & $\begin{array}{c}\text { Thm. } 2.4 .2 \\
-\end{array}$ & $\begin{array}{c}\text { Ex. } 2.1 .2 \\
-\end{array}$ & $\begin{array}{l}\text { (iv) } \\
+\end{array}$ \\
\hline $\begin{array}{l}\text { can be almost } \\
\text { extended to } \mathbf{R}\end{array}$ & $\mathrm{N} / \mathrm{A}$ & ? & $\begin{array}{c}\text { Fact } 2.4 .1 \\
+\end{array}$ & $\begin{array}{c}\text { (iv) } \\
+\end{array}$ \\
\hline
\end{tabular}

(i) A function $f$ from $A \subset \mathbf{R}$ to $\mathbf{R}$ is measurable (or has the Baire property) if $f$ is the restriction of some measurable $F: \mathbf{R} \rightarrow \mathbf{R}$ (having the Baire property) to the set $A$. This is the same as saying that the preimages under $f$ of open sets are intersections of measurable sets (sets with the Baire property) with $A$. As 
an example here take the function $f$ from Example 2.1.2 where $G_{1}$ and $G_{2}$ are of the full outer measure (nowhere meager). Then $f$ is nonmeasurable (does not have the Baire property).

(ii) The function $\chi_{(0, \infty)}$ on $\mathbf{R} \backslash\{0\}$ is an example here.

(iii) Take $f$ as in Example 2.1.2 with $G_{1}$ and $G_{2}$ nowhere meager and of the full outer measure.

(iv) Extend $f$ putting 0 on $\mathbf{R} \backslash A$.

\subsection{Continuity of symmetrically continuous func- tions}

It is always a fundamental question how some other type of continuity is related to the ordinary continuity. Although symmetric continuity is weaker than continuity, symmetrically continuous functions on "nice" domains must be continuous at many points. In this section we are going to investigate the set of points of continuity of symmetrically continuous functions defined on arbitrary subsets of $\mathbf{R}$. Our main results here are Theorem 2.2.7 and Corollaries 2.2.8 and 2.2.9, which are strengthenings of earlier known theorems, quoted below, for functions from $\mathbf{R}$ to $\mathbf{R}$. The proofs have been shifted to the next section as they are long and technical.

The first result that we state was published by Stein and Zygmund in 1960 .

Theorem 2.2.1 (Stein, Zygmund [SZ]) If $f: \mathbf{R} \rightarrow \mathbf{R}$ is symmetrically continuous and measurable then it is continuous everywhere except on a set of measure zero and first category. 
Later on Pesin and Preiss showed that the measurability assumption may be dropped.

Theorem 2.2.2 (Pesin [Pe], Preiss [Pr]) If $f: \mathbf{R} \rightarrow \mathbf{R}$ is symmetrically continuous then $f$ is measurable.

So the obvious corollary says that symmetric continuity implies continuity almost everywhere (in the sense of measure and category).

A more general formulation in the category sense of this corollary is an old theorem of Fried from 1937 (which uses a method of Charzyński).

Theorem 2.2.3 (Fried [Fr]) Let $f: \mathbf{R} \rightarrow \mathbf{R}$ be symmetrically continuous on a residual set. Then $f$ is continuous at every point except on a set of the first category.

Stein and Zygmund had also a stronger result saying that measurability and symmetric continuity only on a measurable set also implies continuity almost everywhere on that set.

Theorem 2.2.4 (Stein, Zygmund [SZ]) If $f: \mathbf{R} \rightarrow \mathbf{R}$ is measurable and symmetrically continuous on a measurable set $E \subset \mathbf{R}$ then $f$ is continuous almost everywhere (in the sense of measure) on $E$.

One may suspect that here, as before, the measurability assumption is not necessary. Indeed this is the case. It follows from the following theorem of Belna from 1983.

Theorem 2.2.5 (Belna [Be]) Let $f: \mathbf{R} \rightarrow \mathbf{R}$ be an arbitrary function. Then the set of points at which $f$ is symmetrically continuous but not continuous has inner measure zero and contains no second category set having the Baire property.

Since for every function from $\mathbf{R} \rightarrow \mathbf{R}$ the set of points of continuity is a $G_{\delta}$ set, which is both measurable and has the Baire property, we have the following corollary. 
Corollary 2.2.6 (Belna [Be]) Let $f: \mathbf{R} \rightarrow \mathbf{R}$ be symmetrically continuous on a set $E$ which is measurable (has the Baire property). Then $f$ is continuous at almost every point of $E$ (on a residual subset of $E$ ).

In the corollary above the function $f$ may of course fail to be even measurable on the set $\mathbf{R} \backslash E$. This raises a question whether we need at all the assumption that the function is defined on the set $\mathbf{R} \backslash E$. The answer is not obvious since the proofs of all the theorems mentioned above use heavily the fact that the function is defined everywhere on $\mathbf{R}$. However, it is indeed enough that the function $f$ is defined only on the set $E$. It follows from the next theorem, which can be regarded as a strengthening of Belna's result (Theorem 2.2.5).

Theorem 2.2.7 Let $f: A \rightarrow \mathbf{R}$, where $A$ is an arbitrary subset of $\mathbf{R}$. Then the set of points at which $f$ is symmetrically continuous but not continuous has the inner measure zero and contains no second category set having the Baire property.

Two corollaries below are also strenghenings of Corollary 2.2.6.

Corollary 2.2.8 Let $E \subset \mathbf{R}$ be measurable and $f: E \rightarrow \mathbf{R}$ be symmetrically continuous. Then $f$ is continuous almost everywhere on $E$.

Corollary 2.2.9 Let $E \subset \mathbf{R}$ have the Baire property and $f: E \rightarrow \mathbf{R}$ be symmetrically continuous. Then $f$ is continuous at every point of $E$ except on a meager set.

In these corollaries the assumption that $E$ is measurable (has the Baire property) cannot be dropped, as Example 2.1.2 shows. It is enough to take groups $G_{1}$ and $G_{2}$ to be dense and at least one of them of positive outer measure. Then $f=\left.\chi_{G_{1}}\right|_{G_{1} \cup G_{2}}$ is symmetrically continuous on its domain but discontinuous everywhere. 
The set $G_{1} \cup G_{2}$ is of course not symmetric. Below we present two much stronger examples with symmetric domains, where a set $A \subset \mathbf{R}$ is symmetric if

$$
\forall_{x \in A} \forall_{h} \quad x+h \in A \Longleftrightarrow x-h \in A .
$$

For example every additive subgroup of $\mathbf{R}$ is symmetric.

Example 2.2.10 Let $A=\left\{\frac{k}{3^{n}}: k \in \mathbf{Z}, n \in \omega\right\}$ and $f: A \rightarrow\{0,1\}$ be defined by

$$
f\left(\frac{k}{3^{n}}\right)= \begin{cases}0 & \text { when } k \text { is even } \\ 1 & \text { when } k \text { is odd. }\end{cases}
$$

Then $f$ is symmetrically continuous and $A$ is symmetric yet $f$ is discontinuous everywhere on its domain.

Proof. The domain $A$ is a group so is obviously symmetric. It is immediate that the symmetric reflection of any point $\frac{k}{3^{n}}$ about a point $\frac{l}{3^{m}}$ preserves the parity of the numerator: $2 \frac{l}{3^{m}}-\frac{k}{3^{n}}=\frac{2 l 3^{n}-k 3^{m}}{3^{m+n}}$. Since both $f^{-1}(0)$ and $f^{-1}(1)$ are dense, $f$ is discontinuous everywhere.

Example 2.2.11 Let $H$ be a Hamel base containing 1 and let $S$ be a linear space over $\mathbf{Q}$ spanned by $H \backslash\{1\}$. Define $A=\{x+k: x \in S, k \in \mathbf{Z}\}$ and $f: A \rightarrow\{0,1\}$ by

$$
f(x+k)= \begin{cases}0 & \text { when } k \text { is even } \\ 1 & \text { when } k \text { is odd }\end{cases}
$$

Then $f$ is symmetrically continuous on $A, A$ is symmetric, and yet $f$ is discontinuous everywhere on $A$.

Proof. Just like in the previous example $A$ is a group, thus symmetric, and $f$ is symmetrically continuous (we preserve the parity of $k$ in reflections).

Example 2.2.11 has many more interesting properties and we will refer to it many times. 


\subsection{Proof of the main result}

We give the proof of Theorem 2.2.7. Theorems 2.3.5 and 2.3.6 are technical and lengthy, but then the proof of Theorem 2.2.7 is short and easy.

We introduce two useful (though technical) definitions.

Definition 2.3.1 A symmetric cover relation on a set $E \subset \mathbf{R}$ is a family $V$ of closed intervals $[a, b]$ such that $\frac{a+b}{2} \in E$.

Definition 2.3.2 If $V$ is a symmetric cover relation on $E \subset \mathbf{R}$ then $V^{5}$ is a family of closed intervals $[a, b]$ such that there is a sequence of points $a=x_{0}, x_{1}, \ldots, x_{5}=b$ such that intervals $\left[x_{i}, x_{i+1}\right]$ belong to $V$ for $i=0,1,2,3,4$. We call the points $x_{1}, \ldots, x_{4}$ the intermediate endpoints.

Uher in [Uh] proved the following theorems (which have been reformulated by Thomson, and we give here Thomson's versions).

Theorem 2.3.3 (Uher [T2, thm. 3.25]) Let $E \subset \mathbf{R}$ have the Baire property and suppose $V$ is a symmetric cover relation on $E$ having the property that for every $x \in E$ there is a positive number $\delta(x)$ so that for every $t \in \mathbf{R}$

$$
0<t<\delta(x) \Rightarrow[x-t, x+t] \in V .
$$

Then there is an open set $G$ such that $E \backslash G$ is of the first category and every interval $[x-t, x+t]$ contained in $G$ belongs to $V^{5}$.

Theorem 2.3.4 (Uher [T2, thm. 3.26]) Let $E \subset \mathbf{R}$ be measurable and suppose $V$ is a symmetric cover relation on $E$ having the property that for every $x \in E$ there is a 
positive number $\delta(x)$ so that for every $t \in \mathbf{R}$

$$
0<t<\delta(x) \Rightarrow[x-t, x+t] \in V .
$$

Then, for almost all points $x \in E$ (in the sense of measure), there is a neighborhood $U_{x}$ of $x$ such that for each $x+t \in U_{x}$ the interval $[x, x+t]$ belongs to $V^{5}$.

We will prove two theorems that are strengthened versions of Uher theorems.

Theorem 2.3.5 Let $E \subset \mathbf{R}$ have the Baire property and suppose $V$ is a symmetric cover relation on $E$ having the property that for every $x \in E$ there is a positive number $\delta(x)$ so that for every $t \in \mathbf{R}$

$$
0<t<\delta(x) \Rightarrow[x-t, x+t] \in V .
$$

Then there is an open set $G$ such that $E \backslash G$ is of the first category and every interval $[x-t, x+t]$ contained in $G$ is in $V^{5}$. Moreover, the intermediate endpoints may be chosen from the set $E$.

Theorem 2.3.6 Let $E \subset \mathbf{R}$ be measurable and suppose $V$ is a symmetric cover relation on $E$ having the property that for every $x \in E$ there is a positive number $\delta(x)$ so that for every $t \in \mathbf{R}$

$$
0<t<\delta(x) \Rightarrow[x-t, x+t] \in V .
$$

Then, for almost all points $x \in E$ (in the sense of measure), there is a neighborhood $U_{x}$ of $x$ such that whenever $x+t \in U_{x}$ then the interval $[x, x+t]$ belongs to $V^{5}$. Moreover, the intermediate endpoints may be chosen from the set $E$. 
In both theorems, the intermediate points may also be chosen to form a monotonic sequence. This property, however, will not be used until Chapter 5 and is easy to take care of. So, it will be skipped in the proofs.

As we see, only the last parts of Theorems 2.3.5 and 2.3.6 make them stronger then their Uher/Thomson's counterparts. We will follow the path of the proof presented in [T2] (attributed to Humke and Laczkovich); unfortunately, our proofs will be more complicated due to the last parts of Theorems 2.3.5 and 2.3.6.

Proof of Theorem 2.3.5. Let $\mathcal{I}$ denote the family of all open intervals $I$ with the property that for every $x, y \in I$ there is a chain $x=x_{0}, x_{1}, \ldots, x_{5}=y$ satisfying

$$
\left[x_{i}, x_{i+1}\right] \in V \text { for } i=0,1,2,3,4 \text { and } x_{i} \in E \text { for } i=1,2,3,4 \text {. }
$$

We will show that

if $E$ is residual in $(a, b)$ then there is a subinterval of $(a, b)$ belonging to $\mathcal{I}$.

The statement of the theorem then follows by taking for the set $G$ a union of a maximal pairwise disjoint subfamily of $\mathcal{I}$. Indeed any interval contained in $G$ is also contained in some interval from $\mathcal{I}$ so it satisfies the assertion of the theorem. To see that $E \backslash G$ is first category note that the set $E$ is residual in some nonempty open set $U$ for which $E \backslash U$ is meager. But then $U$ consists of disjoint intervals $(a, b)$ and $E$ is residual in each of them. So $G$ is dense in $U$ and, since it is open, it is residual in $U$. Thus $E \backslash G \subset(E \backslash U) \cup(U \backslash G)$ is meager as claimed.

To show (2.1) assume that $E$ is residual in $(a, b)$. For any positive $n \in \omega$ let

$$
E_{n}=\left\{x \in E: 0<t<\frac{1}{n} \Rightarrow[x-t, x+t] \in V\right\} .
$$


Then $E=\bigcup_{n=1}^{\infty} E_{n}$ and there is a number $n \in \omega$ so that $E_{n}$ is of second category in $(a, b)$. Thus there is an interval $I \subset(a, b)$ such that $E_{n}$ is of second category in every subinterval of $I$. Assume additionally that the length $|I|$ of $I$ is less than $\frac{1}{n}$. Take the interval $I^{\prime}$ concentric with $I$ and of the length $\frac{1}{10}|I|$. This is this interval $I^{\prime}$ that, as we shall show, belongs to $\mathcal{I}$.

Let $x, y \in I^{\prime}, x<y$. For the sake of simplicity assume that $x=0$. For $x \neq 0$ we repeat the construction below with the set $E$ replaced by $E-x$. Note that for any numbers $t$ with $|t|<\frac{3}{2}\left|I^{\prime}\right|=\frac{3}{20}|I|$ and $r$ with $\frac{1}{2} \leq|r| \leq 2$,

the set $r E_{n}+t$ is second category in every subinterval of $I^{\prime}$.

To see this note that since $0 \in I^{\prime},[-0.9|I|, 0.9|I|] \subset I$. Since $|r| \geq \frac{1}{2}$ we have $[-0.45|I|, 0.45|I|] \subset r I$ and since $|t| \leq \frac{3}{2}\left|I^{\prime}\right|=0.15|I|,[-0.3|I|, 0.3|I|] \subset r I+t$. We see that the interval $I^{\prime}$ is contained in every interval $r I+t$. Since $E_{n}$ is of second category in every subinterval of $I$ then so is $r E_{n}+t$ in $r I+t$.

Similarly,

$$
\text { the sets } \pm \frac{1}{2} E+t \text { are residual in } I^{\prime} \text { for }|t|<\frac{3}{2}\left|I^{\prime}\right|
$$

We want to find a 5 -element chain $0=x_{0}, x_{1}, \ldots, x_{5}=y$ satisfying $(*)$. To do this we will find an interval $J \subset\left(\frac{9}{10} y, y\right)$ such that for every $z \in J$ there is a four element chain $0=x_{0}, x_{1}, \ldots, x_{4}=z$ satisfying

$$
\left[x_{i}, x_{i+1}\right] \in V \text { for } i=0,1,2,3 \text { and } x_{i} \in E \text { for } i=1,2,3 \text {. }
$$

This will finish the proof since then we select $z \in J$ such that $z$ is also in the set $Z=\left\{z \in\left(\frac{9}{10} y, y\right): z \in E\right.$ and $\left.[z, y] \in V\right\}$. The intersection of $J$ and $Z$ is nonempty 
since $\left(2 E_{n}-y\right) \cap E \cap\left(\frac{9}{10} y, y\right) \subset Z\left(\right.$ as $z \in 2 E_{n}-y$ implies $\frac{z+y}{2} \in E_{n}$ so $\left.[z, y] \in V\right)$ and, by (2.2), $2 E_{n}-y$ is second category in every subinterval of $I^{\prime}$.

In the interval $\left(\frac{9}{10} y, y\right)$ the set $E$ is residual and, by $(2.2), E_{n}+\frac{3}{4} y$ is second category since $\frac{3}{4} y<\frac{3}{2}\left|I^{\prime}\right|$. Hence $E \cap\left(E_{n}+\frac{3}{4} y\right)$ is also second category in $\left(\frac{9}{10} y, y\right)$. It follows, from the fact that $E=\bigcup_{n=1}^{\infty} E_{n}$, that there is an integer $m$ and an open interval $J \subset\left(\frac{9}{10} y, y\right)$ so that the set $E_{m} \cap\left(E_{n}+\frac{3}{4} y\right)$ is second category in every subinterval of $J$. We may assume that $|J|<\frac{1}{m}$.

To show that $J$ satisfies (**) pick $z \in J$. By (2.3) the set $\frac{1}{2} E+\frac{3}{4} y$ is residual in $J$ and, by (2.2), the set $\frac{1}{2} E_{n}+\frac{1}{4} z+\frac{3}{8} y$ is second category in $J$ since $\frac{3}{4} y, \frac{1}{4} z+\frac{3}{8} y<y<\left|I^{\prime}\right|$. Therefore we may choose a point

$$
t \in\left(\frac{1}{2} E+\frac{3}{4} y\right) \cap\left(\frac{1}{2} E_{n}+\frac{1}{4} z+\frac{3}{8} y\right) \cap J .
$$

Since $t \in \frac{1}{2} E+\frac{3}{4} y$, we have

$$
q=2 t-\frac{3}{2} y \in E .
$$

Now we choose a point $s$ satisfying

$$
s \in\left(\frac{1}{2} E+\frac{3}{4} y\right) \cap\left(-\frac{1}{2} E+2 t-\frac{3}{4} y\right) \cap\left(\frac{1}{2} E+\frac{1}{2} z\right) \cap\left[E_{m} \cap\left(E_{n}+\frac{3}{4} y\right)\right] \cap J
$$

and $|s-t|<\frac{1}{2} \delta(q)$.

Such a point may be found since, by (2.3), the first three sets are residual in $J$, and the fourth one is of second category in every subinterval of $J$ so their intersection is dense in $J$. Denote by $p$ the point $p=s-\frac{3}{4} y$ and note that

$$
|q-2 p|=2|s-t|<\delta(q) \text { and } p \in E_{n}
$$

Now define our points $x_{i}$ : 


$$
\begin{aligned}
& x_{0}=0=x, \\
& x_{1}=2 p=2 s-\frac{3}{2} y \in E \text { since } 2\left(\frac{1}{2} E+\frac{3}{4} y\right)-\frac{3}{2} y=E, \\
& x_{2}=2 q-2 p=4 t-\frac{3}{2} y-2 s \in E \text { since } 4 t-\frac{3}{2} y-2\left(-\frac{1}{2} E+2 t-\frac{3}{4} y\right)=E, \\
& x_{3}=2 s-z \in E \text { since } 2\left(\frac{1}{2} E+\frac{1}{2} z\right)-z=E, \\
& x_{4}=z .
\end{aligned}
$$

So the second part of $(* *)$ is satisfied.

The centers of $\left[x_{i}, x_{i+1}\right]$ are:

$$
\begin{aligned}
& \frac{x_{0}+x_{1}}{2}=p \in E_{n} \text { by }(2.5), \\
& \frac{x_{1}+x_{2}}{2}=q \in E \text { by }(2.4), \\
& \frac{x_{2}+x_{3}}{2}=2 t-\frac{3}{4} y-\frac{1}{2} z \in E_{n} \text { since } t \in \frac{1}{2} E_{n}+\frac{1}{4} z+\frac{3}{8} y, \\
& \frac{x_{3}+x_{4}}{2}=s \in E_{m} .
\end{aligned}
$$

To finish the proof we only need to verify that each $\left[x_{i}, x_{i+1}\right]$ is in $V$. But this can be seen as follows. Intervals $\left[x_{0}, x_{1}\right]$ and $\left[x_{2}, x_{3}\right]$ are in $V$ since their centers are in $E_{n}$ and each $x_{i}$ is in the interval $I$ which has the length $<\frac{1}{n}$. The interval $\left[x_{1}, x_{2}\right] \in V$ since, by (2.5), $\left|x_{1}-\frac{x_{1}+x_{2}}{2}\right|=|2 p-q|<\delta(q)$. And finally $\left[x_{3}, x_{4}\right]$ belongs to $V$ because $\left|x_{4}-s\right|=|z-s|<\frac{1}{m}$ as both $s, z \in J$ which has length less than $\frac{1}{m}$.

In the proof of the next theorem we use the following notions and facts. By $|A|$ we denote the outer (Lebesgue) measure of the set $A, d(A)$ denotes the set of points of (outer) density of $A$, i.e., the set $\left\{x \in \mathbf{R}: \lim _{h \rightarrow 0^{+}} \frac{|A \cap[x-h, x+h]|}{2 h}=1\right\}$. The 
well known theorem of Lebesgue (see e.g. [T2, thm. A.11]) says that for any set $A$ almost all points of $A$ are its points of density. $d(A)$ is always a measurable set (even for nonmeasurable $A)$ and $d(d(A))=d(A)$. If $x \in d(A)$ then $a x+b \in d(a A+b)$ for $a, b \in \mathbf{R}, a \neq 0$. In particular if $A$ consists only of its density points then so does $a A+b$ for $a, b \in \mathbf{R}, a \neq 0$. We also use the following lemma.

Lemma 2.3.7 [T2, thm. A.11] If sets $A_{n} \subset \mathbf{R}$, where $n \in\{1,2, \ldots, k\}$, are all measurable except at most one of them and $z$ is a point of density of each of them then $z$ is also a point of density of their intersection. In particular in every neighborhood of $z$ there is a point of $\bigcap_{n=1}^{k} A_{n}$.

Proof of Theorem 2.3.6. The proof follows the pattern of the proof of thm. 2.3.5; we will refer to the conditions $(*)$ and $(* *)$ from that proof.

We assume throughout the proof that every measurable set consists only of its density points. Let

$$
E_{n}=\left\{x \in E: 0<t<\frac{1}{n} \Rightarrow[x-t, x+t] \in V\right\} .
$$

We show that every point that belongs to the set $\bigcup_{n=1}^{\infty} d\left(E_{n}\right)$ has a neighborhood satisfying the assertion of the theorem. Thus almost every point of $E$ satisfies it since $E \backslash \bigcup_{n=1}^{\infty} d\left(E_{n}\right)$ is of measure 0 .

Let $x$ be a point of density of $E_{n}$. Assume for notational convenience that $x=0$ (for if $x \neq 0$ then replace the set $E$ by $E-x$ ). There is a number $\eta, 0<\eta<\frac{1}{n}$, such that for every $y$ with $|y|<\eta$ and every $t$ with $|t|<2|y|$ each of the sets

$$
2 E_{n}+t, \pm \frac{1}{2} E_{n}+t, \frac{3}{2} E_{n}+t, \text { and } E \cap\left(E_{n}+t\right)
$$


intersects the interval $\left(\frac{9}{10} y, y\right)$ in a set of outer measure greater than $\frac{9}{100}|y|$. We may take $\eta=\frac{1}{6} \delta$, where $\delta$ is such that for every $0<h<\delta$ we have $\frac{\left|E_{n} \cap[-h, h]\right|}{2 h} \geq$ $\frac{999}{1000}$. To see that this $\eta$ works take for example the set $\frac{1}{2} E_{n}+t$ and note that $\left(\frac{1}{2} E_{n}+t\right) \cap(0.9 y, y)=\frac{1}{2}\left[E_{n} \cap(1.8 y-2 t, 2 y-2 t)\right]+t$. Numbers $|1.8 y-2 t|$ and $|2 y-2 t|$ are less than $6|y|<\delta$, so

$$
\begin{aligned}
\left|E_{n} \cap(1.8 y-2 t, 2 y-2 t)\right|= & \mid E_{n} \cap(-6 y, 6 y) \\
& \backslash\left[E_{n} \cap[(-6 y, 6 y) \backslash(1.8 y-2 t, 2 y-2 t)]\right] \mid \\
\geq & \frac{999}{1000} 12|y|-(12|y|-0.2|y|) \\
= & \frac{188}{1000}|y|>2 \frac{9}{100}|y| .
\end{aligned}
$$

The calculations for other sets in (2.6) are similar.

Fix any $y \in(0, \eta)$. We prove that there is a chain $0=x_{0}, x_{1}, \ldots, x_{5}=y$ satisfying $(*)$. A similar argument would work for $y<0$.

Let $Z=\left\{z \in\left(\frac{9}{10} y, y\right):[z, y] \in V\right\}$. Note that $\left(2 E_{n}-y\right) \cap\left(\frac{9}{10} y, y\right) \subset Z$ and so, by (2.6), $|Z| \geq \frac{9}{100} y$. To prove the theorem it is enough to show that there is a measurable set $F \subset\left(\frac{9}{10} y, y\right) \cap E$ such that $|F|>\frac{1}{100} y$ and that for every $z \in F$ there is a four element chain $0=x_{0}, \ldots, x_{4}=z$ satisfying $(* *)$. Indeed if this were true then $F \cap Z \neq \emptyset$ and choosing any $z \in F \cap Z$ we would obtain a four element chain which expanded by $x_{5}=y$ would give us our desired chain.

Recall that, by (2.6), $\left.\mid E \cap\left(E_{n}+\frac{3}{4} y\right)\right) \cap\left(\frac{9}{10} y, y\right) \mid>\frac{9}{100} y$. So, from the fact that $E$ is an increasing sum of $E_{m}$ 's, there is an integer $m$ so that

$$
\left|E_{m} \cap\left(E_{n}+\frac{3}{4} y\right) \cap\left(\frac{9}{10} y, y\right)\right|>\frac{8}{100} y .
$$


Denote $A_{m}=E_{m} \cap\left(E_{n}+\frac{3}{4} y\right) \cap\left(\frac{9}{10} y, y\right)$ and write

$$
F=\left(\frac{1}{2} E+\frac{3}{4} y\right) \cap d\left(\frac{2}{3} E_{n}+\frac{1}{2} y\right) \cap d\left(A_{m}\right) \cap E .
$$

We show that all points of $F$ have the property claimed for it.

First note that, by (2.6) and (2.7),

$$
|F|>\frac{1}{10} y-\frac{1}{100} y-\frac{1}{100} y-\frac{2}{100} y-\frac{1}{100} y>\frac{5}{100} y .
$$

Let $z \in F$. So $z$ is a density point of $F$. Since $z \in d\left(\frac{2}{3} E_{n}+\frac{1}{2} y\right)$ it follows that $z$ belongs to $d\left(\frac{1}{2} E_{n}+\frac{1}{4} z+\frac{3}{8} y\right)$ (indeed $\frac{3}{4} z \in d\left(\frac{3}{4}\left(\frac{2}{3} E_{n}+\frac{1}{2} y\right)\right)$ so $\frac{1}{4} z+\frac{3}{4} z \in$ $\left.d\left(\frac{3}{4}\left(\frac{2}{3} E_{n}+\frac{1}{2} y\right)+\frac{1}{4} z\right)\right)$, and since $z \in E, z \in \frac{1}{2} E+\frac{1}{2} z$.

We may pick a point $t$ so that

$$
t \in\left(\frac{1}{2} E_{n}+\frac{1}{4} z+\frac{3}{8} y\right) \cap\left(\frac{1}{2} E+\frac{1}{2} z\right) \cap\left(\frac{1}{2} E+\frac{3}{4} y\right) \cap d\left(A_{m}\right)
$$

and $|z-t|<\frac{1}{m}$.

This is possible by Lemma 2.3.7 since all sets above, except possibly the first one, are measurable and the point $z$ is a density point of each of them.

We define $q=2 t-\frac{3}{2} y$ and note that $q \in E$ since $2\left(\frac{1}{2} E+\frac{3}{4} y\right)-\frac{3}{2} y=E$. Note also that $t$ is a density point of the set $-\frac{1}{2} E+2 t-\frac{3}{4} y$ as $t \in \frac{1}{2} E+\frac{3}{2} y$.

We now pick a point $s$. We want $s$ to satisfy:

$$
\begin{gathered}
s \in\left(\frac{1}{2} E+\frac{1}{2} z\right) \cap\left(\frac{1}{2} E+\frac{3}{4} y\right) \cap\left(-\frac{1}{2} E+2 t-\frac{3}{4} y\right) \cap A_{m}, \\
|s-t|<\frac{1}{2} \delta(q), \text { and }|s-z|<\frac{1}{m} .
\end{gathered}
$$

Once again by Lemma 2.3.7 there exists a point $s$ being in all four sets and arbitrary close to $t$ ( $t$ is a density point of each of the above set). Taking $s$ close 
enough to $t$ we guarantee the last condition since $|s-z|<|z-t|+|t-s|$ and $|z-t|<\frac{1}{m}$.

Define the point $p=s-\frac{3}{4} y$ and note that $|q-2 p|=2|s-t|<\delta(q)$. The points $x_{0}, x_{1}, \ldots, x_{4}$ satisfying $(* *)$ are defined as follows:

$$
\begin{aligned}
& x_{0}=x=0, \\
& x_{1}=2 p=2 s-\frac{3}{2} y \in E \text { since } 2\left(\frac{1}{2} E+\frac{3}{4} y\right)-\frac{3}{2} y=E, \\
& x_{2}=2 q-2 p=4 t-2 s-\frac{3}{2} y \in E \text { since } 4 t-\frac{3}{2} y-2\left(-\frac{1}{2} E+2 t-\frac{3}{4} y\right)=E, \\
& x_{3}=2 s-z \in E \text { since } 2\left(\frac{1}{2} E+\frac{1}{2} z\right)-z=E, \\
& x_{4}=z .
\end{aligned}
$$

The centers of the intervals $\left[x_{i}, x_{i+1}\right]$ are:

$$
\begin{aligned}
& \frac{x_{0}+x_{1}}{2}=p=s-\frac{3}{4} y \in E_{n} \text { since } s \in A_{m} \subset E_{n}+\frac{3}{4} y . \\
& \frac{x_{1}+x_{2}}{2}=q=2 t-\frac{3}{2} y \in E \text { since } t \in \frac{1}{2} E+\frac{3}{4} y . \\
& \frac{x_{2}+x_{3}}{2}=2 t-\frac{3}{4} y-\frac{1}{2} z \in E_{n} \text { since } t \in \frac{1}{2} E_{n}+\frac{1}{4} z+\frac{3}{8} y . \\
& \frac{x_{3}+x_{4}}{2}=s \in E_{m} \text { since } s \in A_{m} \subset E_{m} .
\end{aligned}
$$

Intervals $\left[x_{0}, x_{1}\right]$ and $\left[x_{2}, x_{3}\right]$ are in $V$ since their centers are in $E_{n}$ and each $x_{i}$ is in the interval $I$ which has the length $<\frac{1}{n}$. The interval $\left[x_{1}, x_{2}\right] \in V$ since $\left|x_{1}-\frac{x_{1}+x_{2}}{2}\right|=\left|x_{1}-q\right|=2|s-t|<\delta(q)$. And finally $\left[x_{3}, x_{4}\right]$ belongs to $V$ because $\left|x_{4}-s\right|=|z-s|<\frac{1}{m}$. This way we see that each interval $\left[x_{i}, x_{i+1}\right]$ belongs to $V$ and the proof is finished. 
Proof of Theorem 2.2.7. Let us denote by $S C_{f}$ the set of points where $f$ is symmetrically continuous and by $C_{f}$ the set of continuity points. We prove only that $S C_{f} \backslash C_{f}$ contains no measurable set of positive measure. The proof that $S C_{f} \backslash C_{f}$ contains no second category set having the Baire property is essentially the same.

Assume that $E \subset S C_{f}$ is measurable. We will show that almost all points in $E$ are also in $C_{f}$. This shows that the only measurable subsets of $S C_{f} \backslash C_{f}$ are of measure zero so $S C_{f} \backslash C_{f}$ has inner measure zero.

Fix an $\varepsilon>0$ and define a symmetric cover relation on $E$ :

$$
V=\{[x-t, x+t]: x \pm t \in E \Rightarrow|f(x-t)-f(x+t)|<\varepsilon\} .
$$

This relation satisfies assumption of Theorem 2.3.6. So for almost every $x \in E$ there is a neighborhood $U_{x}$ so that $x+t \in U_{x} \Rightarrow[x, x+t] \in V^{5}$ and intermediate endpoints are in $E$, that is, there are points $x_{0}=x, x_{1}, \ldots, x_{5}=x+t$ such that $x_{i} \in E$ for $i=1,2,3,4$ and $\left[x_{i}, x_{i+1}\right] \in V$ for $i=0, \ldots, 4$. Therefore $|f(x)-f(x+t)|<5 \varepsilon$. Since $\varepsilon$ has been chosen arbitrarily, $f$ is continuous in almost all points of $E$.

\subsection{Extensions of continuous and symmetrically continuous functions}

In this section we look at the extension properties of symmetrically continuous functions and compare them with these properties for continuous functions.

We know that every continuous function $f: A \rightarrow \mathbf{R}$ defined on a subset of $\mathbf{R}$ can be extended to a continuous function defined on a dense $G_{\delta}$ set. This set can be of course residual. Note that continuity distinguishes between measure and category as 
it is possible to find function that can be only extended to a measure zero $G_{\delta}$ set. $^{1}$ We will see in Theorem 2.4.2 that some symmetrically continuous functions cannot be extended beyond measure zero and first category domain.

If we look at Example 2.2.11 we see that a symmetrically continuous function defined there has dense preimages of 0 and 1 , so any its extension would be discontinuous at every point. Thus, by Corollaries 2.2.8 and 2.2.9, $f$ cannot be extended to a symmetrically continuous function defined on a measurable set or on a set having the Baire property.

The function $f$ from Example 2.2.11 cannot be even "almost extended" (in a sense that the extension differs from $f$ only on a set of measure 0 ) to a symmetrically continuous function. This follows from the fact that $A$, and thus the sets $f^{-1}(1)$ and $f^{-1}(0)$, are nonmeasurable (note that all rational translations of $f^{-1}(1)$ and $f^{-1}(0)$ cover $\mathbf{R}$ so these sets have positive outer measure, in fact full outer measure). So even upon removing sets of measure zero from them they remain dense in $\mathbf{R}$.

A different question is whether having a function $f: A \rightarrow \mathbf{R}$ that is continuous (or symmetrically continuous) we may extend it to the whole real line leaving it continuous (or symmetrically continuous) on the set $A$. For continuous functions, as the next theorem shows, this is true. (The fact below must have been known, but we were unable to find the references.)

Fact 2.4.1 If $f: A \rightarrow \mathbf{R}$ is continuous then there is an extension $\hat{f}$ of $f$ defined on

\footnotetext{
${ }^{1}$ An example of such function may be built the following way. Let $C_{n} \subset[0,1]$ be a ternary Cantor set such that its compliment $G_{n}$ has measure $\frac{1}{n}$. Define function $f_{n}: G_{n} \rightarrow \mathbf{R}$ such that its restriction to each component interval of $G_{n}$ is a linear function with range $(0,1)$. Obviously $f_{n}$ is continuous on $G_{n}$ and has variation 1 at every point outside $G_{n}$. Now, the function $f=\sum_{n \in \omega} \frac{1}{3^{n}} f_{n}$ defined on $\bigcap_{n \in \omega} G_{n}$ is a desired function.
} 
the whole $\mathbf{R}$ with $A \subset C_{\hat{f}}$, where $C_{\hat{f}}$ is the set of points of continuity of $\hat{f}$.

Proof. If $A$ is not dense in $\mathbf{R}$ then $\mathbf{R} \backslash \operatorname{cl}(A)$ consists of disjoint intervals $(a, b)$ and we extend our function by defining $f$ on each of them as a continuous function joining $\sup _{x \rightarrow a, x \in A} f(x)$ with $\sup _{x \rightarrow b, x \in A} f(x)$ (even if one of them or both are infinity). So we may assume that $A$ is dense in $\mathbf{R}$.

Assume first that $f$ is bounded. In this case we may assume that $f: A \rightarrow[0,1]$. Take a closure $\operatorname{cl}(f)$ of the function $f$ in $\mathbf{R}^{2}(f$ is a set of pairs thus a subset of $\left.\mathbf{R}^{2}\right)$. Since $A$ is dense, the projection $\pi(\operatorname{cl}(f))$ of $\operatorname{cl}(f)$ onto the first coordinate is the whole real line. This is so, as for every finite interval $[a, b]$, the $\operatorname{set} \operatorname{cl}\left(\left.f\right|_{[a, b]}\right)$ is compact and its projection is also compact, thus contains $A \cap[a, b]$ - a dense set in $[a, b]$.

Let $I_{x}=\{y:\langle x, y\rangle \in \operatorname{cl}(f)\}$. Then every $I_{x}$ is nonempty. Also, for every $x \in A$ the set $I_{x}$ is the one element set $\{f(x)\}$. Indeed, for every $\left\{\left\langle x_{n}, f\left(x_{n}\right)\right\rangle\right\}$ in $f$ with $\left\{x_{n}\right\}$ converging to $x$, we have $\lim f\left(x_{n}\right)=f(x)$ by the continuity of $f$. Take an indexed selector $S$ of the family $\left\{I_{x}: x \in \mathbf{R}\right\}$ (i.e., $S$ is a set of pairs $\langle x, y\rangle$, where $\left.y \in I_{x}\right)$. Then $S$ is a function from $\mathbf{R}$ to $[0,1]$ and $S$ agrees with $f$ on every $x \in A$. It is easy to check that $S$ is continuous in every point of $A$.

If $f$ is unbounded then take the function $g=\arctan$ of, which is bounded. By the continuity of arctan we have that $g$ is continuous in every point where $f$ is continuous.

Having an extension $\hat{g}$ of $g$, we come back to $f$ taking $\hat{f}=\tan \circ \hat{g}$, where we define $\hat{f}\left( \pm \frac{\pi}{2}\right)=0$. Once again by the continuity of the function tan we preserve points of continuity of $\hat{g}$. 
Once again the translation of the above theorem into symmetrical language fails to hold.

Theorem 2.4.2 There is a function $f: A \rightarrow\{0,1\}$ that is symmetrically continuous everywhere on $A$ with the following property: if $F$ is an extension of $f$ to a domain $D$ such that $D$ has positive measure or $D$ is of second category with the Baire property, then $F$ is not symmetrically continuous at some point on $A$.

Proof. We will construct inductively (using transfinite induction) a set $A$ and will define function $f$ on $A$. Function $f$ will have two properties: for every perfect set $P$, there is a point $x \in P$ such that there is a pair of sequences $\left\{y_{n}\right\}$ and $\left\{z_{n}\right\}$ in $A$ converging to $x$ and symmetric about $x$, (i.e., $\frac{y_{n}+z_{n}}{2}=x$ for all $n$ ); $f$ will have value 1 for all $y_{n}^{\prime} s$ and value 0 for all $z_{n}^{\prime} s$. Then, of course, $x$ cannot belong to the domain of any symmetrically continuous extension of $f$ and, since any measurable set of positive measure or Baire measurable set of second category contains a perfect set, $f$ cannot be extended to any of these sets.

Let $\left\{P_{\alpha}: \alpha<\mathbf{c}\right\}$ be a list of all perfect subsets of $\mathbf{R}$. Take any point $x_{0} \in P_{0}$ and define $y_{n}^{0}=x_{0}-\frac{1}{n}$ and $z_{n}^{0}=x_{0}+\frac{1}{n}$. Put also $f_{0}:\left\{y_{n}^{0}, z_{n}^{0}: n \in \omega\right\} \rightarrow\{0,1\}, f_{0}\left(y_{n}^{0}\right)=1$, $f_{0}\left(z_{n}^{0}\right)=0$. (We do not define $f_{0}\left(x_{0}\right)$.)

Since $x_{0}$ is the only accumulation point of the set $\left\{y_{n}^{0}, z_{n}^{0}: n \in \omega\right\}$, the function $f_{0}$ is symmetrically continuous (vacuously) on its domain. Denote by $T_{0}$ the linear space over $\mathbf{Q}$ spanned by the set $\left\{y_{n}^{0}, z_{n}^{0}: n \in \omega\right\}$ and note that $x_{0} \in T_{0}$.

Assume that for $\beta<\alpha$ we have defined points $x_{\beta} \in P_{\beta}$ and sequences $\left\{y_{n}^{\beta}\right\}$ and $\left\{z_{n}^{\beta}\right\}$ converging to $x_{\beta}$ and $\frac{y_{n}^{\beta}+z_{n}^{\beta}}{2}=x_{\beta}$. Assume also that $x_{\beta}, y_{n}^{\beta}, z_{n}^{\beta} \notin T_{\beta}$ where $T_{\beta}=$ linear space over $\mathbf{Q}$ spanned by the set $\left\{x_{\gamma}, y_{n}^{\gamma}, z_{n}^{\gamma}: n \in \omega, \gamma<\beta\right\}$ (i.e. the points 
$x_{\beta}, y_{n}^{\beta}, z_{n}^{\beta}$ are independent of the previous ones).

Functions $f_{\beta}:\left\{y_{n}^{\beta}, z_{n}^{\beta}: n \in \omega\right\} \rightarrow\{0,1\}$ are defined like $f_{0}$, that is, $f_{\beta}\left(y_{n}^{\beta}\right)=1$ and $f_{\beta}\left(z_{n}^{\beta}\right)=0$. (We leave $f_{\beta}\left(x_{\beta}\right)$ not defined.)

Let $T_{\alpha}$ be the linear space over $\mathbf{Q}$ spanned by the set $\left\{x_{\beta}, y_{n}^{\beta}, z_{n}^{\beta}: n \in \omega, \beta<\alpha\right\}$. The space $T_{\alpha}$ has cardinality $|\alpha| \times \omega<\mathbf{c}$ and we may find a point $x_{\alpha} \in P_{\alpha} \backslash T_{\alpha}$ and sequences $\left\{y_{n}^{\alpha}\right\},\left\{z_{n}^{\alpha}\right\}$ disjoint with $T_{\alpha}$, converging to $x_{\alpha}$, and with $\frac{x_{n}^{\alpha}+z_{n}^{\alpha}}{2}=x_{\alpha}$ for all $n \in \omega$. Like before we define $f_{\alpha}\left(y_{n}^{\alpha}\right)=0$ and $f_{\alpha}\left(z_{n}^{\alpha}\right)=1$. Again $f_{\alpha}$ is (vacuously) symmetrically continuous on its domain.

Having our construction done for all $\alpha<\mathbf{c}$ we define $f=\bigcup_{\alpha<\mathbf{c}} f_{\alpha}$. Since points $y_{n}^{\alpha}$ and $z_{n}^{\alpha}$ are linearly independent of the points $y_{n}^{\beta}$ and $z_{n}^{\beta}$ for $\alpha \neq \beta$, the function $f$ is vacuously symmetrically continuous. Putting $A=\operatorname{dom}(f)=\left\{y_{n}^{\alpha}, z_{n}^{\alpha} ; n \in \omega, \alpha<\mathbf{c}\right\}$ we have constructed the desired set and the function defined on it.

Is is worth to add that we may strengthen the theorem above by adding that $A$ may be of measure zero and first category. (We may select points $y_{n}^{\alpha}$ and $z_{n}^{\alpha}$ from the set $C$ constructed in Corollary 3.1.9.)

Note also that in contrast to this theorem the function $f$ in Example 2.2.11 can be extended to $\mathbf{R}$ (as could be any symmetrically continuous function defined on a symmetric set, by putting 0 on $\mathbf{R} \backslash A$ ), and remain symmetrically continuous in every point of $A$. 


\section{Chapter 3}

\section{Weak symmetric continuity}

\section{1}

The results in this chapter are in my paper [Sz1].

Let us recall a known fact about points of continuity. (See e.g. [K].)

Fact 3.1.1 The set of points of continuity of any function (from $\mathbf{R}$ to $\mathbf{R}$ ) is a $G_{\delta}$ set and any $G_{\delta}$ set is the set of points of continuity for some function.

Many authors have investigated what happens if we replace the ordinary continuity by other types of continuity, i.e., which sets may be obtained as sets of points of different types of generalized continuity. First let us look at the symmetric continuity. This is a weaker notion than ordinary continuity and Fact 3.1.1 is not valid any more for points of symmetric continuity. In fact it is an old problem of Marcus to characterize these points. (We mentioned already this problem in Chapter 2 and stated related Theorem 2.1.3. For other partial results see Thomson [T2] and Jaskuła, Szkopińska [JS].)

Another notion we consider is weak continuity. 
Definition 3.1.2 A function $f: \mathbf{R} \rightarrow \mathbf{R}$ is weakly continuous at a point $x$ if there exist the sequences $\left\langle x_{n}\right\rangle$ and $\left\langle y_{n}\right\rangle$ such that $x_{n} \nearrow x, y_{n} \searrow x$, and

$$
\lim _{n \rightarrow \infty} f\left(x_{n}\right)=\lim _{n \rightarrow \infty} f\left(y_{n}\right)=f(x) .
$$

In particular $f\left(x_{n}\right)-f\left(y_{n}\right) \rightarrow 0$.

The next, rather unexpected, theorem says that weak continuity is indeed a very weak notion.

Theorem 3.1.3 [T1, Chap. 2] Every function $f: \mathbf{R} \rightarrow \mathbf{R}$ is weakly continuous everywhere except a countable set and every countable subset of $\mathbf{R}$ is the set of points of weak discontinuity for some function.

Proof. For the first part see for example [T1, Chap. 2].

For the second part, let $A=\left\{a_{n}: n \in \omega\right\}$ be a countable set. The function $f(x)=\sum_{a_{n}<x} 2^{-n}$ is the one we are looking for. Indeed, if $x>a_{n}>y$ then $f(x)-f(y) \geq 2^{-n}$, so $f$ is not weakly continuous at any $a_{n}$. If $x \notin A$ and if $y$ is closer to $x$ than any of the points $a_{1}, \ldots, a_{k}$ then $|f(y)-f(x)| \leq \sum_{n=k+1}^{\infty} 2^{-n}=2^{-k}$ since we do not have any $a_{n}$ 's in $(x-|x-y|, x+|x-y|)$ with $n \leq k$. So $f$ is continuous (so weakly continuous) at $x \notin A$.

Now if we replace weak continuity by its symmetric counterpart, we obtain the following notion.

Definition 3.1.4 A function $f: \mathbf{R} \rightarrow \mathbf{R}$ is weakly symmetrically continuous at a point $x$ if there exists a sequence $\left\langle h_{n}\right\rangle$ converging to 0 such that

$$
\lim _{n \rightarrow \infty} f\left(x+h_{n}\right)-f\left(x-h_{n}\right)=0
$$


(Paradoxically, weak symmetric continuity is a stronger notion than weak continuity.) We will investigate the problem of characterizing the sets of points where a function can be weakly symmetrically continuous. The first result in this direction was obtained by Ciesielski and Larson, and it looks surprising in light of Theorem 3.1.3.

Theorem 3.1.5 (Ciesielski, Larson [CL]) There is a function $g: \mathbf{R} \rightarrow \mathbf{N}$ that is weakly symmetrically discontinuous everywhere.

The next theorem answers the problem completely.

Theorem 3.1.6 Any set of reals is the set of points of weak symmetrical continuity for some function $f: \mathbf{R} \rightarrow \mathbf{N}$.

The main part of the proof is in the following lemma. We will use the Axiom of Choice here. It cannot be avoided as there are models of ZF (see [CL, cor. 1.5]) in which Lemma 3.1.7 and Theorem 3.1.5 are false.

Lemma 3.1.7 For any set $A \subset \mathbf{R}$ there exists a set $X=\bigcup_{a \in A}\left\{a \pm h_{n}^{a}: n \in \omega\right\}$ such that

(a) $h_{n}^{a} \searrow 0$ for every $a \in A$ and the sets $S_{a}=\left\{a \pm h_{n}^{a}: n \in \omega\right\}$ are pairwise disjoint;

(b) for every $b \in \mathbf{R} \backslash A$ there is no sequence $h_{n} \searrow 0$ with $b \pm h_{n} \in X$.

Before we start the proof let us remark that the set $X$ may be always chosen to be meager and of measure zero. This partially answers the question stated at the Miniconference in Auburn in 1997 after presentation of Lemma 3.1.7. The set $X$ can be found as a subset of some special meager and measure zero set $C$. To 
make the below proof work, we need only that for all $x \in \mathbf{R}$ and for all $n \in \omega$ there are continuum many pairs $y, z \in C \cap\left(x-\frac{1}{n}, x+\frac{1}{n}\right)$ with $\frac{y+z}{2}=x$. Corollary 3.1.9 guarantees the existence of such set $C$ (this is this set $C$ that the comment after Theorem 2.4.2 refers to). In the proof below however, for the sake of simplicity (and since we do not need that strengthened version to prove Theorem 3.1.6), we take $C=\mathbf{R}$. The reader may notice that with no changes the proof works for other sets $C$.

Proof of Lemma 3.1.7. We will construct the set $X$ inductively, using transfinite induction. First note that the first part (a) is equivalent to the following condition

(a') For every $a \in A$ there are the sequences $\left\langle x_{n}\right\rangle$ and $\left\langle y_{n}\right\rangle$ from $X$ such that $x_{n} \nearrow a$, $y_{n} \searrow a$, and $\frac{x_{n}+y_{n}}{2}=a$ for every $n$.

Condition (b) than says that there are no such sequences $\left\langle x_{n}\right\rangle,\left\langle y_{n}\right\rangle$ for $b \notin A$.

Let $\kappa$ be the cardinality of $A$ and let $A=\left\{a_{\alpha}: \alpha<\kappa\right\}$ be an enumeration of

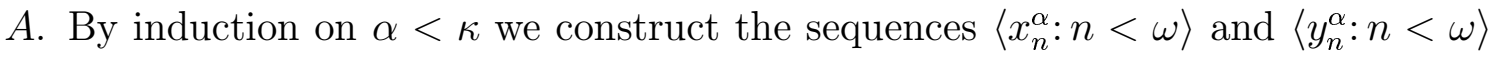
witnessing $\left(\mathrm{a}^{\prime}\right)$ for $a=a_{\alpha}$, aiming for $X=\bigcup_{\alpha<\kappa}\left\{x_{n}^{\alpha}, y_{n}^{\alpha}: n<\omega\right\}$. The challenge will be in maintaining condition (b). We will use the following auxiliary sets:

$$
\begin{gathered}
X_{\alpha}=\bigcup_{\beta<\alpha}\left(\left\{x_{n}^{\beta}: n<\omega\right\} \cup\left\{y_{n}^{\beta}: n<\omega\right\}\right), \\
B_{\alpha}=\left\{\frac{x+y}{2}: x, y \in X_{\alpha}\right\} \backslash A,
\end{gathered}
$$

and the functions $f_{\alpha}: B_{\alpha} \rightarrow \mathbf{R}$ defined by

$$
f_{\alpha}(b)=\inf \left\{|b-x|: x \in X_{\alpha} \text { and } \exists_{y \in X_{\alpha}} \frac{x+y}{2}=b\right\} .
$$

In the construction we will maintain the following two inductive conditions: 
(c) $f_{\alpha}(x)>0$ for each $x \in B_{\alpha}$,

(d) $f_{\beta} \subset f_{\alpha}$ for $\beta \leq \alpha$.

Conditions (c) and (d) imply that (b) holds for every point in $\cup B_{\alpha}$, since $f=\cup_{\alpha<\kappa} f_{\alpha}$ is positive on $\cup B_{\alpha}$.

For every point outside $\bigcup_{\alpha<\kappa} B_{\alpha}$, condition (b) is automatically satisfied and the set $X$ will have the desired property.

Take the first sequence $\left\langle x_{n}^{0}\right\rangle$ to be $x_{n}^{0}=a_{0}+\frac{1}{n}$. Then $y_{n}^{0}=a_{0}-\frac{1}{n}, X_{0}=$ $\left\{x_{n}^{0}, y_{n}^{0}: n<\omega\right\}$, and $B_{0}=\left\{\frac{x+y}{2}: x, y \in X_{0}\right\} \backslash A$. Since the only accumulation point of $X_{0}$ is $a_{0} \in A$ then $f_{0}(x)>0$ for all $x \neq a_{0}$ and (c) is satisfied.

Assume that for some $\alpha<\kappa$ we have already defined the sequences $\left\langle x_{n}^{\beta}\right\rangle$ and $\left\langle y_{n}^{\beta}\right\rangle$ for all $\beta<\alpha$. We choose sequences $\left\langle x_{n}^{\alpha}\right\rangle$ and $\left\langle y_{n}^{\alpha}\right\rangle$ satisfying ( $\left.\mathrm{a}^{\prime}\right)$ so that for every $b \in \bigcup_{\beta<\alpha} B_{\beta}$ and $n<\omega$ we have

$$
x_{n}^{\alpha}, 2 b-x_{n}^{\alpha} \notin \bigcup_{\beta<\alpha} X_{\beta} \cup\left\{x_{k}^{\alpha}, y_{k}^{\alpha}: k<n\right\}
$$

and

$$
x_{n}^{\alpha}, 2 b-y_{n}^{\alpha} \notin \bigcup_{\beta<\alpha} X_{\beta} \cup\left\{x_{k}^{\alpha}, y_{k}^{\alpha}: k<n\right\} .
$$

Such sequences exist since we may choose points $x_{n}^{\alpha}$ and $y_{n}^{\alpha}$ inductively so that

$$
x_{n}^{\alpha}, y_{n}^{\alpha} \notin\left\{2 b-x: b \in \bigcup_{\beta<\alpha} B_{\beta} \& x \in\left(\bigcup_{\beta<\alpha} X_{\beta} \cup\left\{x_{k}^{\alpha}, y_{k}^{\alpha}: k<n\right\}\right)\right\}
$$

(this set has cardinality $\left|\bigcup_{\beta<\alpha} B_{\beta}\right| \otimes\left|\bigcup_{\beta<\alpha} X_{\beta}\right| \leq|\alpha| \otimes \omega<\mathbf{c}$ ) and $\frac{x_{n}^{\alpha}+y_{n}^{\alpha}}{2}=a_{\alpha}$. It is clear that both inductive conditions are preserved.

Now putting $X=\bigcup_{\alpha<\kappa} X_{\alpha}=\bigcup_{\alpha<\kappa}\left\{x_{n}^{\alpha}, y_{n}^{\alpha}: n<\omega\right\}$, we see that (a) is obviously satisfied and (b) is satisfied since $f=\bigcup_{\alpha<\kappa} f_{\alpha}$ is a positive function and for every 
point $b$ not in $A$ either we do not have a pair of sequences contained in $X$ and symmetric about that point or these sequences can not have elements closer to $b$ than $f(b)$.

Proof of Theorem 3.1.6. Let $g: \mathbf{R} \rightarrow \mathbf{N}$ be an everywhere weakly symmetrically discontinuous function and $A \subset \mathbf{R}$. We will construct a function $f$ that has points of weak symmetric continuity exactly in the set A. Let X be the set from Lemma 3.1.7 for the set A. We define

$$
f(x)=\left\{\begin{array}{cc}
g(x)+1 & \text { for } x \notin X \\
0 & \text { for } x \in X .
\end{array}\right.
$$

Obviously every point of $\mathrm{A}$ is a point of weak symmetric continuity of $f$. (There is a symmetric sequence in $\mathrm{X}$ converging to this point.) If $x \notin A$ then we do not have a sequence symmetrically convergent to $x$ contained in $X$. We also do not have such a sequence contained in $\mathbf{R} \backslash X$ satisfying the definition of weak symmetric continuity for $x$, because $g$ is weakly symmetrically discontinuous everywhere. Finally any "mixed" sequence will not satisfy that definition either since $f$ differ on points from $X$ and outside of $X$ by at least 1 .

Now we provide the proof of the existence of measure zero and meager set $C$. This set can be used both in Lemma 3.1.7 and in Theorem 2.4.2. The main theorems of this work do not require that set, and the reader may skip that part. We include this since it seems that it is worth adding that "small" sets can have big "weak symmetric closure."

We will use the base 7 expansion, i.e., for $x \in[0,1]$ we write $x=0 . x_{1} x_{2} \ldots$ meaning that $x=\sum_{i=1}^{\infty} \frac{x_{i}}{7^{2}}, x_{i} \in\{0,1,2,3,4,5,6\}$. 
For every finite sequence $x_{1} x_{2} \ldots x_{n}$ where $x_{i} \in\{0,1,2,3,4,5,6\}$ let

$$
C_{x_{1} x_{2} \ldots x_{n}}=\left\{x \in[0,1]: x=0 . x_{1} x_{2} \ldots x_{n} x_{n+1} \ldots x_{i} \neq 3 \text { for } i>n\right\} .
$$

$C_{x_{1} x_{2} \ldots x_{n}}$ is a "Cantor $\frac{1}{7}$ " set. It is a perfect set of measure zero (thus, nowhere dense).

Denote also

$$
\hat{C}=\bigcup\left\{C_{x_{1} x_{2} \ldots x_{n}}: n \in \omega\right\} .
$$

The set $\hat{C}$ being a countable union of measure zero and nowhere dense sets has measure zero and is meager. The next theorem says it is the set we are looking for. We say that a set $A \subset \mathbf{R}$ is symmetric about a point $x \in \mathbf{R}$ if $x+h \in A \Longleftrightarrow$ $x-h \in A$ for every $h \in \mathbf{R}$ (the same as $A=2 x-A$ ).

Theorem 3.1.8 For every $x \in(0,1)$ and for every open neighbourhood $U$ of $x$ there is a perfect set $P$ contained in $\hat{C} \cap U$ and symmetric about $x$.

Proof. Let $x \in(0,1)$ and let $0 . x_{1} x_{2} \ldots$ be the expansion of $x$. We assume that not almost all $x_{i}^{\prime} s$ are equal to 6 . Let also $U$ be an arbitrary neighbourhood of $x$. We fix a number $n \in \omega$ such that for all $y=0 . y_{1} y_{2} \ldots$ with $y_{i}=x_{i}$ for $i \leq n$ we have $y \in U$.

We consider three cases.

Case 1. In the sequence $x_{1} x_{2} \ldots$ we have infinitely many 1's or 2's or 3's or 4's or 5's.

Say at first that we have infinitely many 1 's and that $x_{i_{j}}=1$ for $j=1,2,3, \ldots$ and $i_{j}>n$. We define numbers $y$ and $z$.

$$
\begin{aligned}
& y=0 . y_{1} y_{2} \ldots \text { and } z=0 . z_{1} z_{2} \ldots \text { where } \\
& y_{i}=x_{i} \text { for } i \leq n, z_{i}=x_{i} \text { for } i \leq n,
\end{aligned}
$$




$$
\begin{aligned}
& y_{i_{j}}=0 \text { or } y_{i_{j}}=1, z_{i_{j}}=2-y_{i_{j}}, \\
& y_{i}=2 \text { if } x_{i}=3, i>n, z_{i}=4 \text { if } x_{i}=3, i>n, \\
& y_{i}=x_{i}, z_{i}=x_{i} \text { for all other } i .
\end{aligned}
$$

Clearly all such $y$ 's form a "Cantor-like" perfect set and so do $z$ 's, every $y$ and $z$ belongs to $U \cap C_{x_{1} x_{2} \ldots x_{n}}$. For every pair of corresponding $y$ and $z$ we have $\frac{y_{i}+z_{i}}{2}=x_{i}$ so $\frac{y+z}{2}=x$.

If we have infinitely many other digits instead then we repeat the above construction changing only $y_{i_{j}}$ and $z_{i_{j}}$ according to the following formulas.

$$
\begin{aligned}
& x_{i_{j}}=2 \rightarrow y_{i_{j}}=0 \text { or } y_{i_{j}}=4, z_{i_{j}}=4-y_{i_{j}}, \\
& x_{i_{j}}=3 \rightarrow y_{i_{j}}=0 \text { or } y_{i_{j}}=6, z_{i_{j}}=6-y_{i_{j}}, \\
& x_{i_{j}}=4 \rightarrow y_{i_{j}}=2 \text { or } y_{i_{j}}=4, z_{i_{j}}=8-y_{i_{j}}, \\
& x_{i_{j}}=5 \rightarrow y_{i_{j}}=5 \text { or } y_{i_{j}}=6, z_{i_{j}}=10-y_{i_{j}} .
\end{aligned}
$$

Once again we see that $\frac{y_{i}+z_{i}}{2}=x_{i}$ so $\frac{y+z}{2}=x$ and that $y$ 's and $z$ 's form a symmetric perfect set around $x$ that is contained in $\hat{C} \cap U$.

Case 2. In $x_{1} x_{2} \ldots$ we have infinitely many 0 's and 6's and Case 1 doesn't hold. In that case we have infinitely many pairs $x_{i}=6, x_{i+1}=0$. Let $x_{i_{j}}=6, x_{i_{j}+1}=0$ for $j=1,2, \ldots$ and $i_{j}>n$.

We define numbers $y=0 . y_{1} y_{2} \ldots$ and $z=0 . z_{1} z_{2} \ldots$.

$$
\begin{aligned}
& y_{i}=x_{i}, z_{i}=x_{i} \text { for } i \leq n, \\
& \left(y_{i_{j}}=6 \text { and } y_{i_{j}+1}=0\right) \text { or }\left(y_{i_{j}}=5 \text { and } y_{i_{j}+1}=1\right), \\
& z_{i_{j}}=6, z_{i_{j}+1}=0 \text { if } y_{i_{j}+1}=0 \text { and } z_{i_{j}+1}=5 \text { if } y_{i_{j}+1}=1, \\
& y_{i}=2 \text { if } x_{i}=3, i>n, z_{i}=4 \text { if } x_{i}=3, i>n, \\
& y_{i}=x_{i}, z_{i}=x_{i} \text { in other places. }
\end{aligned}
$$

We may easily check that $\frac{y+z}{2}=x$ (since $\frac{y_{i}+z_{i}}{2}=x_{i}$ ), $y$ 's and $z$ 's are in $U$ and in 
$C_{x_{1} x_{2} \ldots x_{n}}$. It's also clear that $y$ 's and $z$ 's form a perfect set.

Case 3. In $x_{1} x_{2} \ldots$ all $x_{i}$ are eventually equal 0.

Now $x=0 . x_{1} x_{2} \ldots x_{k} 000 \ldots$ and $x_{k} \neq 0$.

If $k>n$ then the set $C_{x_{1} x_{2} \ldots\left(x_{k}-1\right)} \cup C_{x_{1} x_{2} \ldots x_{k}}$ is a desired set.

If $k \leq n$ then the set $C_{x_{1} x_{2} \ldots\left(x_{k}-1\right) 66 \ldots 6} \cup C_{x_{1} x_{2} \ldots x_{k} 00 \ldots 0}(n-k 0$ 's and $n-k 6$ 's) is a right set.

Now if we have a copy of $\hat{C}$ in every interval $[n, n+1](n \in \mathbf{Z})$ that is if

$$
C=\bigcup_{n \in \mathbf{Z}} \hat{C}+n
$$

then Theorem 3.1.8 holds for any $x \in \mathbf{R}$, and we have the corollary.

Corollary 3.1.9 The set $C$ is of measure zero and first category and for every $x \in \mathbf{R}$ and every open neighbourhood $U$ of $x$ there is a perfect set $P$ contained in $C \cap U$ and symmetric about $x$.

In Theorem 3.1.6 we built a function with infinitely many values. An interesting problem that Ciesielski and Larson ask in [CL] is whether this is necessary, that is, if there is a nowhere weakly symmetrically continuous function with finitely many values.

In case of 2-valued function we have the following result. (Compare also [CL].)

Theorem 3.1.10 (Nowik [N]) Every 2-valued function has only countably many points of weak symmetric discontinuity.

Ciesielski in [Ci1] showed also that every 3-valued function has a point of weak symmetric continuity. We do not know if the same is true for 4 -valued functions. Recently 
Ciesielski and Shelah [CSh] showed that there is everywhere weakly symmetrically discontinuous function with bounded range. 


\section{Chapter 4}

\section{"Bad" symmetrically continuous functions}

\subsection{Introduction}

This chapter concerns the "irregularity" of symmetrically continuous functions. While they are continuous almost everywhere (Theorems 2.2.1, 2.2.2, and 2.2.3), they still can fail to satisfy some other (quite weak) generalized continuity properties. (See [T1, Ch. 3, 70-84] or [T2].) In this chapter we will follow our paper [CSz].

For $X \subset \mathbf{R}$ a function $f: X \rightarrow \mathbf{R}$ is countably continuous if there is a countable cover $\left\{X_{n}: n \in \mathbf{N}\right\}$ of $X$ (by arbitrary sets) such that each restriction $f \mid X_{n}$ is continuous.

An interesting question asked by Lee Larson (private communication) is whether every symmetrically continuous function is countably continuous. The main aim of this chapter is to give a negative answer to this question.

We will use the following notion. For $X \subset \mathbf{R}$ a function $f: X \rightarrow \mathbf{R}$ is said to be of Sierpiński-Zygmund type if $f \mid Y$ is discontinuous for every $Y \subset X$ of cardinality c, the cardinality of $\mathbf{R}$. 
The following fact describes the basic relation between these classes of functions.

Fact 4.1.1 Let $f: \mathbf{R} \rightarrow \mathbf{R}$. If there exists $X \subset \mathbf{R}$ of cardinality $\mathbf{c}$ such that $f \mid X$ is of Sierpinski-Zygmund type then $f \mid X$ and $f$ are not countably continuous.

Proof. If $\left\{X_{n} \subset X: n \in \mathbf{N}\right\}$ is a cover of $X$ then there exists $n \in \mathbf{N}$ such that $X_{n}$ has cardinality $\mathbf{c}$. (Since the cofinality of $\mathbf{c}$ is uncountable.) Thus, $f \mid X_{n}$ is discontinuous, as $f \mid X$ is of Sierpiński-Zygmund type.

\subsection{Technical Lemmas}

The construction presented below is, in a big part, based on the technique developed by Chlebík in [Ch]. In particular, the next four lemmas are the modifications of their counterparts from [Ch].

The set of centers of symmetry of a set $A \subset \mathbf{R}$ will be denoted by $A^{\star}$, i.e.,

$$
A^{\star}=\{x \in \mathbf{R}:(\forall k \in \mathbf{R})(x+k \in A \Longleftrightarrow x-k \in A)\} .
$$

For any function $f: X \rightarrow \mathbf{R}$ with $X \subset \mathbf{R}$ the symbol $C(f)$ will stand for the set of continuity points of $f$ and $D(f)$ for the set of points of discontinuity. Thus, $D(f)=\mathbf{R} \backslash C(f)$.

Lemma 4.2.1 Let $h: \mathbf{R} \rightarrow \mathbf{R}$ be symmetrically continuous and $\left\{A_{\alpha}\right\}_{\alpha \in \mathcal{A}}$ be a family of disjoint subsets of $\mathbf{R}$ such that for every $x \in \mathbf{R}$

$$
\lim _{y \rightarrow x} h(y)=0 \quad \text { or } \quad x \in \bigcap_{\alpha \in \mathcal{A}} A_{\alpha}^{\star} .
$$

If $r_{\alpha} \in[0,1]$ for every $\alpha \in \mathcal{A}$ then the function

$$
f=h \cdot \sum_{\alpha \in \mathcal{A}} r_{\alpha} \chi_{A_{\alpha}}
$$


is symmetrically continuous.

Proof. This is a modification of [Ch, Lemma 2]. First note that $0 \leq \sum_{\alpha \in \mathcal{A}} r_{\alpha} \chi_{A_{\alpha}} \leq 1$ since the sets $A_{\alpha}$ are disjoint. Let $x \in \mathbf{R}$.

If $\lim _{y \rightarrow x} h(y)=0$ then

$$
\lim _{y \rightarrow x} f(y)=\lim _{y \rightarrow x}\left(h \cdot \sum_{\alpha \in \mathcal{A}} r_{\alpha} \chi_{A_{\alpha}}\right)(y)=0
$$

since $\sum_{\alpha \in \mathcal{A}} r_{\alpha} \chi_{A_{\alpha}}$ is bounded. Hence $f$ is symmetrically continuous at $x$.

If $x \in \bigcap_{\alpha \in \mathcal{A}} A_{\alpha}^{\star}$ then $\sum_{\alpha \in \mathcal{A}} r_{\alpha} \chi_{A_{\alpha}}(x-k)=\sum_{\alpha \in \mathcal{A}} r_{\alpha} \chi_{A_{\alpha}}(x+k)$ for every $k \in \mathbf{R}$, and so

$$
\lim _{k \rightarrow 0} f(x+k)-f(x-k)=\lim _{k \rightarrow 0}[h(x+k)-h(x-k)] \sum_{\alpha \in \mathcal{A}} r_{\alpha} \chi_{A_{\alpha}}(x+k)=0 .
$$

Thus, once again, $f$ is symmetrically continuous at $x$.

Lemma 4.2.2 If $G \subset \mathbf{R}$ is an additive subgroup then $G \subset(2 G+x)^{\star}$ for every $x \in G$.

Proof. Let $g \in G, a \in 2 G+x$, and $b$ be a point symmetric to $a$ with respect to $g$, i.e., such that $a+b=2 g$. We have to prove that $b \in 2 G+x$.

So, let $z \in G$ be such that $a=2 z+x$. Then

$$
b=2 g-a=2 g-(2 z+x)=2(g-z-x)+x \in 2 G+x
$$

since $g, x, z \in G$.

Lemma 4.2.3 If $G \subset \mathbf{R}$ is an additive subgroup then the sets $\{2 G+x: x \in G\}$ form a partition of $G$. 
Proof. This is just the usual coset decomposition of $G$ using the subgroup $2 G$. To see it explicitly, assume that for some $x, y \in G$ there exists $z \in(2 G+x) \cap(2 G+y)$. We have to prove that $2 G+x=2 G+y$, i.e., that $x-y \in 2 G$. So, let $a, b \in G$ be such that $z=2 a+x=2 b+y$. Then $x-y=2(b-a) \in 2 G$.

Lemma 4.2.4 There exists a symmetrically continuous function $h: \mathbf{R} \rightarrow \mathbf{R}$ with the property that

(i) $C(h)=h^{-1}(0)$,

(ii) $D(h)$ is an additive subgroup of $\mathbf{R}$, and

(iii) there exists a subset $X$ of $D(h)$ of cardinality $\mathbf{c}$ such that

$$
(2 D(h)+x) \cap(2 D(h)+y)=\emptyset \text { for every distinct } x, y \in X .
$$

Proof. Chlebík in [Ch, thm.1] constructed a symmetrically continuous function $h: \mathbf{R} \rightarrow \mathbf{R}$ satisfying (i) ${ }^{1}$ and (ii) for which there exists a set $X \subset D(h)$ of cardinality c with the property that

$$
2 D(h)+H_{1} \neq 2 D(h)+H_{2} \text { for every distinct } H_{1}, H_{2} \subset X \text {. }
$$

In particular, if $x \in X$ and $y \in X$ are distinct and $H_{1}=\{x\}, H_{2}=\{y\}$, then

$$
2 D(h)+x=2 D(h)+H_{1} \neq 2 D(h)+H_{2}=2 D(h)+y .
$$

So, by Lemma 4.2.3, $(2 D(h)+x) \cap(2 D(h)+y)=\emptyset$.

\footnotetext{
${ }^{1}$ Chlebík remarks only that $h=\lim _{m \rightarrow \infty}\left(1+\sum_{n=1}^{m}\left|\frac{1}{n} \sin 3^{n} x\right|\right)^{-1}$ is continuous at every point of $h^{-1}(0)$, implying that $h^{-1}(0) \subset C(h)$. However $h^{-1}(0)$ is clearly dense in $\mathbf{R}$. (For example for all $x$ 's in the form $\pi\left(m+\sum_{i=1}^{\infty} \frac{a_{i}}{3^{i}}\right)$, where $m \in \mathbf{Z}$ and the numbers $a_{i} \in\{0,1,2\}$ are eventually equal 1.)
} 


\subsection{Main result}

Theorem 4.3.1 There exists a symmetrically continuous function $f: \mathbf{R} \rightarrow \mathbf{R}$ and a subset $X$ of $\mathbf{R}$ of cardinality $\mathbf{c}$ such that $f \mid X$ is of a Sierpinski-Zygmund type.

Proof. Let

$$
\mathcal{D}=\left\{\langle G, g\rangle: G \text { is a } G_{\delta} \text { subset of } \mathbf{R} \text { and } g: G \rightarrow \mathbf{R} \text { is continuous }\right\}
$$

and let $\left\{\left\langle G_{\alpha}, g_{\alpha}\right\rangle: \alpha<\mathbf{c}\right\}$ be an enumeration of $\mathcal{D}$. Also, let $h: \mathbf{R} \rightarrow \mathbf{R}$ and $X$ be from Lemma 4.2.4. Pick a one-to-one enumeration $\left\{x_{\alpha}: \alpha<\mathbf{c}\right\}$ of $X$.

By transfinite induction on $\alpha<\mathbf{c}$ define a sequence of real numbers $\left\{r_{\alpha}: \alpha<\mathbf{c}\right\}$ such that the following inductive condition is satisfied for every $\alpha<\mathbf{c}$ :

$$
r_{\alpha} \in[0,1] \backslash\left\{\frac{g_{\beta}\left(x_{\alpha}\right)}{h\left(x_{\alpha}\right)}: \beta \leq \alpha \& x_{\alpha} \in G_{\beta}\right\} .
$$

(Note that $h\left(x_{\alpha}\right) \neq 0$ since $x_{\alpha} \in D(h)=\mathbf{R} \backslash h^{-1}(0)$.)

Now let $A_{\alpha}=2 D(h)+x_{\alpha}$ for every $\alpha<\mathbf{c}$ and notice that, by Lemma 4.2 .4 , the sets $\left\{A_{\alpha}: \alpha<\mathbf{c}\right\}$ are disjoint. Define

$$
f=h \cdot \sum_{\alpha<\mathbf{c}} r_{\alpha} \chi_{A_{\alpha}}
$$

Thus, $f$ is a well defined real function. Moreover, by Lemma 4.2.2,

$$
D(h) \subset \bigcap_{\alpha<\mathbf{c}}\left(2 D(h)+x_{\alpha}\right)^{\star}=\bigcap_{\alpha<\mathbf{c}} A_{\alpha}^{\star},
$$

since $D(h)$ is an additive subgroup of $\mathbf{R}$. So, by Lemma 4.2.1, $f$ is symmetrically continuous, since $\mathbf{R} \backslash D(h)=C(h)=h^{-1}(0)$, implying (4.1). It remains to show that is $f \mid X$ is of Sierpiński-Zygmund type. 
For this, by way of contradiction, assume that there exists $Y \subset X$ of cardinality c such that $f \mid Y$ is continuous. Then there exists a $G_{\delta}$ set $G \subset \mathbf{R}$ containing $Y$ and a continuous function $g: G \rightarrow \mathbf{R}$ such that $g|Y=f| Y$. In particular, $\langle g, G\rangle \in \mathcal{D}$, and there exists a $\beta<\mathbf{c}$ such that $\langle g, G\rangle=\left\langle g_{\beta}, G_{\beta}\right\rangle$. Also, since $Y$ has cardinality $\mathbf{c}$, there exists an $\alpha<\mathbf{c}, \alpha \geq \beta$, such that $x_{\alpha} \in Y$. But $h\left(x_{\alpha}\right) \neq 0$, since $x_{\alpha} \in X \subset$ $D(h)=\mathbf{R} \backslash C(h)=\mathbf{R} \backslash h^{-1}(0)$. So, by (4.2) and the fact that $x_{\alpha} \in A_{\alpha}$,

$$
f\left(x_{\alpha}\right)=h\left(x_{\alpha}\right) \cdot r_{\alpha} \neq h\left(x_{\alpha}\right) \frac{g_{\beta}\left(x_{\alpha}\right)}{h\left(x_{\alpha}\right)}=g_{\beta}\left(x_{\alpha}\right)=g\left(x_{\alpha}\right)
$$

contradicting $g|Y=f| Y$.

Corollary 4.3.2 There is a symmetrically continuous function $f: \mathbf{R} \rightarrow \mathbf{R}$ which is not countably continuous.

Proof. By Theorem 4.3.1 and Fact 4.1.1.

Notice also that in Fact 4.1.1 and Corollary 4.3.2 we can conclude also that $f$ is not $\kappa$-continuous (the graph of $f$ cannot be covered by the graphs of $\kappa$ many continuous functions) where $\kappa$ is less then the cofinality of $\mathbf{c}$.

It is also worth to mention that neither Chlebík's theorem nor Corollary 4.3.2 can be generalized for the class of symmetrically differentiable functions, i.e., the functions $f: \mathbf{R} \rightarrow \mathbf{R}$ for which the limit

$$
\lim _{k \rightarrow 0} \frac{f(x+k)-f(x-k)}{2 k}
$$

exists and is finite for every $x \in \mathbf{R}$. This follows from Theorem 5.1.2, since the set $D(f)$ for every such function is at most countable. On the other hand, we do not know whether the same is true if we allow the limit (4.3) to be infinite. 


\subsection{Continuity cardinal}

For a cardinal number $\kappa$ we will write $\operatorname{cf}(\kappa)$ for its cofinality. The functions are identified with their graphs. The class of all functions from $\mathbf{R}$ into $\mathbf{R}$ is denoted by $\mathbf{R}^{\mathbf{R}}$. The class of all continuous functions is denoted by $\mathcal{C}$. By Sc we denote the family of all symmetrically continuous functions.

Definition 4.4.1 For any family of functions $\mathcal{F} \subset \mathbf{R}^{\mathbf{R}}$ we define $\operatorname{dec}(\mathcal{F}, \mathcal{C})$ as the minimal cardinal number $\kappa$ such that every $f \in \mathcal{F}$ can be covered by $\kappa$ many continuous functions. Or, equivalently, that $\mathbf{R}$ can be partitioned into $\kappa$ many subsets $X_{\alpha}$ and $f \mid X_{\alpha}$ is continuous for every $\alpha<\kappa$.

In this section we will present the proof of the following theorem

Theorem 4.4.2 (Ciesielski, Szyszkowski, see $[\mathrm{Ci} 4]) \operatorname{dec}(\operatorname{Sc}, \mathcal{C})=\operatorname{dec}\left(\mathbf{R}^{\mathbf{R}}, \mathcal{C}\right)$.

This shows that symmetrically continuous functions can be quite "irregular" despite the fact that they are continuous almost everywhere.

More interesting facts have been proven in [Ci4]. The following two theorems will not be used later but they shed a new light on Theorem 4.4.2.

Theorem 4.4.3 $\operatorname{dec}(\mathrm{SZ}, \mathcal{C})=\operatorname{dec}\left(\mathbf{R}^{\mathbf{R}}, \mathcal{C}\right)$.

Theorem 4.4.4 (a) It is consistent with $Z F C$ that $\operatorname{cf}(\mathbf{c})<\mathbf{c}=\operatorname{dec}\left(\mathbf{R}^{\mathbf{R}}, \mathcal{C}\right)$.

(b) It is consistent with ZFC that $\operatorname{dec}\left(\mathbf{R}^{\mathbf{R}}, \mathcal{C}\right)=\operatorname{cf}(\mathbf{c})<\mathbf{c}$.

In order to prove Theorem 4.4.2 we will show the following fact that is of interests by its own. 
Proposition 4.4.5 There exists a perfect set $P \subset \mathbf{R}$ with the property that for every $f_{0}: P \rightarrow[0,1]$ there is a symmetrically continuous function $f: \mathbf{R} \rightarrow \mathbf{R}$ extending $f_{0}$.

Proof. We apply here Lemmas 4.2.1 and 4.2.4. The proof is a compilation of the results contained in $[\mathrm{CSz}]$ and $[\mathrm{Ch}]$. We must take a closer look at Lemma 4.2.1. Namely, we want to show that the set $X$ defined there can contain a perfect set.

Towards the construction of the set $X$ from Lemma 4.2.4 Chlebík in [Ch, thm. 1] defines the following. He takes an arbitrary linear basis $\mathcal{H} \subset(0,1]$ of $\mathbf{R}$ over $\mathbf{Q}$ with $1 \in \mathcal{H}$, puts $\Lambda=\mathcal{H} \backslash\{1\}$, and defines $X=\pi \cdot \psi[\Lambda]$ for a continuous injection $\psi:(0,1] \backslash \mathbf{Q} \rightarrow(0,1)$ given by the formula

$$
\psi(x)=\sum_{k=1}^{\infty} \mu_{k} \cdot 3^{-\left(2^{k}+1\right)},
$$

where $x=\sum_{k=1}^{\infty} \mu_{k} \cdot 2^{-k}$, with $\mu_{k} \in\{0,1\}$, is the unique binary representation of $x$.

To prove Proposition 4.4.5 we need also two additional facts that

(iv) $h$ is of Baire class one (as upper semicontinuous), and that

(v) $X$ is a continuous image of $\mathcal{H} \backslash\{1\}$, where $\mathcal{H}$ is an arbitrary linear basis of $\mathbf{R}$ over $\mathbf{Q}$ with $1 \in \mathcal{H} \subset(0,1]$.

Now, take a perfect set $K \subset[0,1]$ which is linearly independent over $\mathbf{Q}$. (See e.g. [Ku, thm. 2, Ch. XI sec. 7].) Decreasing it, if necessary, we can assume that 1 is linearly independent of $K$. (If it is not, take a finite subset $A$ of $K$ which spans 1 and replace $K$ by its perfect subset disjoint with $A$.) Thus, there exists a linear basis $\mathcal{H} \subset(0,1]$ of $\mathbf{R}$ over $\mathbf{Q}$ such that $\{1\} \cup K \subset \mathcal{H}$. In particular, $X_{0}=\psi[K]$, where $\psi$ is defined in (4.4), is a perfect subset of $X=\psi[\mathcal{H} \backslash\{1\}]$, so it satisfies (iii) from Lemma 4.2.4. 
Now, $h \mid X_{0}$ has a point of continuity, say $x_{0} \in X_{0}$, since $h$ is of Baire class one. (See e.g. [Br].) Since, $x_{0} \in X \subset D(h)=\mathbf{R} \backslash h^{-1}(0)$ we have $h\left(x_{0}\right) \neq 0$. Thus, we can take a perfect subset $P$ of $X_{0}$ for which $h[P] \subset[b, 1]$ for some $b>0$. We will show that $P$ satisfies Proposition 4.4.5.

To see it note that by Lemma 4.2.1 used with $X=P$ the function

$$
f(x)=\frac{1}{b} h(x) \cdot \sum_{y \in P} r(y) \chi_{2 D(h)+y}(x)
$$

is symmetrically continuous for any function $r: P \rightarrow[0,1]$. Moreover, $f(x)=\frac{1}{b} h(x)$. $r(x)$ for every $x \in P$ since $x \in 2 D(h)+x$. ( $D(h)$ is a group.) Thus defining $r$ by

$$
r(x)=\frac{b}{h(x)} \cdot f_{0}(x) \leq f_{0}(x)
$$

we obtain that $r: P \rightarrow[0,1]$ and

$$
f(x)=\frac{1}{b} h(x) \cdot r(x)=f_{0}(x)
$$

for every $x \in P$. This finishes the proof of Proposition 4.4.5.

Proof of Theorem 4.4.2. Clearly $\operatorname{dec}(\operatorname{Sc}, \mathcal{C}) \leq \operatorname{dec}\left(\mathbf{R}^{\mathbf{R}}, \mathcal{C}\right)$. To prove the other inequality take an arbitrary $g \in \mathbf{R}^{\mathbf{R}}$ and let $\kappa=\operatorname{dec}(\{g\}, \mathcal{C})$. It is enough to prove that

$$
\kappa \leq \operatorname{dec}(\operatorname{Sc}, \mathcal{C}) .
$$

If $\kappa<\omega_{1}$ than (4.5) follows from $\operatorname{cf}(\mathbf{c}) \leq \operatorname{dec}(\mathrm{Sc}, \mathcal{C})$. So, we can assume that $\kappa$ is uncountable. Also, if $\varphi$ is a homeomorphism between $\mathbf{R}$ and $(0,1)$ then it is easy to see that $\operatorname{dec}(\{g\}, \mathcal{C})=\operatorname{dec}(\{\varphi \circ g\}, \mathcal{C})$. So, we can assume that $g: \mathbf{R} \rightarrow$ $(0,1)$. Moreover, if $\mathcal{N}=\mathbf{R} \backslash \mathbf{Q}$ then $\kappa=\operatorname{dec}(\{g\}, \mathcal{C})=\operatorname{dec}(\{g \mid \mathcal{N}\}, \mathcal{C})$, since $\kappa$ is uncountable. 
Let $\varphi$ be a homeomorphism between $\mathcal{N}$ and a subset $M$ of $P$, where $P$ is from Proposition 4.4.5, and define $f_{0}: M \rightarrow[0,1]$ by $f_{0}=g \circ \varphi^{-1}$. Once again it is easy to see that $\kappa=\operatorname{dec}(\{g\}, \mathcal{C})=\operatorname{dec}\left(\left\{f_{0}\right\}, \mathcal{C}\right)$. Now, if $f$ is a symmetrically continuous function extending $f_{0}$, which exists by Proposition 4.4.5, then

$$
\kappa=\operatorname{dec}\left(\left\{f_{0}\right\}, \mathcal{C}\right) \leq \operatorname{dec}(\{f\}, \mathcal{C}) \leq \operatorname{dec}(\operatorname{Sc}, \mathcal{C})
$$

proving (4.5). The proof of Theorem 4.4.2 is complete. 


\section{Chapter 5}

\section{Symmetric derivative}

\subsection{Symmetric and ordinary derivatives}

Following the ordinary analysis, symmetric analysis studies also symmetric differentiation. In many cases the symmetric theory resembles the ordinary differentiation theory on the real line. Here we present some aspects of symmetric differentiation, stressing in particular symmetric differentiation for the functions defined on subsets of the real line.

As expected, the symmetric derivative is defined as follows.

Definition 5.1.1 We say that a function $f: \mathbf{R} \rightarrow \mathbf{R}$ is symmetrically differentiable at a point $x \in \mathbf{R}$ if the limit

$$
\lim _{h \rightarrow 0} \frac{f(x+h)-f(x-h)}{2 h}
$$

exists and is finite. (We sometimes allow also infinite values.) The value of this limit is called the symmetric derivative of $f$.

Of course the symmetric differentiability is stronger then symmetric continuity. An old theorem of Charzyński states how one condition implies another. 
Theorem 5.1.2 (Charzyński $[\mathrm{T} 2$, thm. 2.9]) Let the function $f$ (defined on the whole real line) satisfy at every point $x$ the condition

$$
\limsup _{h \rightarrow 0}\left|\frac{f(x+h)-f(x-h)}{2 h}\right|<\infty .
$$

Then the set of points of discontinuity of $f$ is countable.

(We will comment on this theorem after introducing Definition 5.1.6.)

To illustrate the resemblance to the ordinary derivative we state a few monotonicity theorems.

Theorem 5.1.3 [T2, cor. 5.2] Let the function $f: \mathbf{R} \rightarrow \mathbf{R}$ have positive symmetric derivative everywhere. Then $f$ is increasing on the set of its points of continuity. More precisely there is an increasing function $g$ such that $f$ agrees with $g$ on its points of continuity.

Theorem 5.1.4 [T2, thm. 5.5] Let the function $f: \mathbf{R} \rightarrow \mathbf{R}$ have positive symmetric derivative everywhere on a measurable set $E$. Then $f$ is locally increasing at almost every point of $E$ (i.e., almost every point $x$ of $E$ has a neighborhood $U$ such that whenever $y<x<z$ and $y, z \in U$ then $f(y)<f(x)<f(z))$.

Theorem 5.1.5 [T2, thm. 5.4] Let the function $f: \mathbf{R} \rightarrow \mathbf{R}$ have positive symmetric derivative on a set $E$ that has the Baire property. Then there is an open set $G$ so that $E \backslash G$ is meager and $f$ is increasing on each component of $G$.

Now, let us turn to functions defined on subsets of $\mathbf{R}$. 
Definition 5.1.6 Let $A \subset \mathbf{R}$. We say that a function $f: A \rightarrow \mathbf{R}$ is symmetrically differentiable at a point $x \in \mathbf{R}$ if the limit

$$
\lim _{h \rightarrow 0} \frac{f(x+h)-f(x-h)}{2 h}
$$

exists and is finite, taking $h$ from the set $\{h \in \mathbf{R}: x \pm h \in A\}$. (We assume that this set has an accumulation point at 0.) We sometimes also allow infinite values.

Not surprisingly, in general case, all theorems for the functions on the whole real line do not hold for the functions defined only on a subset of $\mathbf{R}$. For example, Theorem 5.1.2 fails, for if we look at Example 2.2.11, we see that there is a function $f$ defined on a symmetric set which has symmetric derivative equal 0 but it is discontinuous everywhere.

Moreover, by Lemma 3.1.7 (used with $A=\mathbf{R}$ ) we have the following fact.

Fact 5.1.7 There is a set $X \subset \mathbf{R}$ such that for any function $f: \mathbf{R} \rightarrow[-\infty, \infty]$ there is a function $g: X \rightarrow \mathbf{R}$ which is symmetrically differentiable and its symmetric derivative is equal to $f$.

(Note that we use here the fact that a function need not be defined at a point to be symmetrically differentiable there.)

Proof. By Lemma 3.1.7 used with $A=\mathbf{R}$ we know that there is a set $X$ which is a union of pairwise disjoint sets $\bigcup_{n<\omega}\left\{x_{n}^{a}, y_{n}^{a}\right\}$, with $a \in \mathbf{R}$, such that the sequences $\left\{x_{n}^{a}\right\}_{n}$ and $\left\{y_{n}^{a}\right\}_{n}$ are convergent to $a$ and symmetric to each other with respect to $a$.

Now defining $g$ on $x_{n}^{a}$ and $y_{n}^{a}$ so that $\frac{g\left(x_{n}^{a}\right)-g\left(y_{n}^{a}\right)}{x_{n}^{a}-y_{n}^{a}} \rightarrow f(\alpha)$ finishes the proof.

Note that the set $X$ may be additionally chosen to be of measure zero and of the first category. This follows from the comment before Lemma 3.1.7. 
Because of such examples we will rather confine ourselves to symmetric domains (except in Theorems 5.1.10, 5.1.9, and 5.2.4). However, even this restriction does not help much. The dramatic failure of Theorems 5.1.4 and 5.1.5 is illustrated by Example 2.2.11. In this example function $f$ is defined on a symmetric set and is symmetrically differentiable with the symmetric derivative being zero everywhere on the domain. Yet $f$ has only values 0 and 1 so is not monotonic in any sense. We may easily "correct" $f$ to have positive derivative (for example by adding the identity function restricted to the domain of $f$ ), and obtain a nonincreasing function.

This example is actually not surprising as even for the ordinary derivative we have the following fact.

Fact 5.1.8 For any function $f: \mathbf{Q} \rightarrow \mathbf{R}$ there is a differentiable function $g: \mathbf{Q} \rightarrow \mathbf{R}$ (with differentiability defined in an obvious way) such that $g^{\prime}=f$.

Proof. Let $\mathbf{Q}=\left\{x_{n}: n \in \omega\right\}$. Define $g_{0}: \mathbf{Q} \rightarrow \mathbf{R}$ by putting $g_{0}(r)=\left(r-x_{0}\right) f\left(x_{0}\right)$ for $\left|r-x_{0}\right|<\sqrt{2}$ and $g_{0}(r)=0$ for $\left|r-x_{0}\right|>\sqrt{2}$.

We define functions $g_{n}: \mathbf{Q} \rightarrow \mathbf{R}$ inductively in the following way. $g_{n}(r)=\left(r-x_{n}\right) f\left(x_{n}\right)+g_{n-1}\left(x_{n}\right)$ for $r$ so close to $x_{n}$ that

- $\left|r-x_{n}\right|<\frac{1}{3^{n}} \sqrt{2} \min \left\{\left|x_{n}-x_{k}\right|: k<n\right\}$,

- $\left|f\left(x_{n}\right)\left(r-x_{n}\right)\right|<\frac{1}{3^{n}} \min \left\{\left|x_{n}-x_{k}\right|: k<n\right\}$,

- $\left|f\left(x_{n}\right)\left(r-x_{n}\right)\right|<\frac{1}{3^{n}}$,

and $g_{n}(r)=g_{n-1}(r)$ for other $r$.

Now, the function $g=\lim g_{n}$ is a desired function. To see this consider for example the point $x_{0}$. The derivative of $g_{0}$ at $x_{0}$ is obviously $f\left(x_{0}\right)$ and every $g_{n}\left(x_{0}\right)=$ 
$g_{0}\left(x_{0}\right)=0$. Fix an $\varepsilon>0$ and find first $n \in \omega$ such that $\left|x_{n}-x_{0}\right|<\varepsilon$. We have $g_{n-1}(r)=g_{0}(r)$ for $r \in\left(x_{0}-\frac{\varepsilon}{2}, x_{0}+\frac{\varepsilon}{2}\right)$. We also have $g_{n-1}(r)=g_{n}(r)$ for $r$ close to $x_{0}$. For $r$ close to $x_{n}$ we have that $g_{n}(r)$ differs from $g_{n-1}(r)=g_{0}(r)$ by at most $\frac{1}{3^{n}} \min \left\{\left|x_{n}-x_{k}\right|: k<n\right\}<\frac{1}{3^{n}}\left|r-x_{0}\right|$ and for these $r$ 's any other $g_{k}$ with $k>n$ differs from $g_{0}(r)$ by not more then $\sum_{k \geq n} \frac{1}{3^{k}}\left|r-x_{0}\right|=\frac{2}{3^{n}}\left|r-x_{0}\right|$. So

$$
\frac{g(r)-g\left(x_{0}\right)}{r-x_{0}}
$$

differs from $f\left(x_{0}\right)$ not more than $\frac{2}{3^{n}}$.

The calculations in any other point $x_{n}$ are the same.

In contrast to the above, when taking the domain which is "big and nice enough," we do have positive results similar to Theorems 5.1.4 and 5.1.5. The following theorems are generalizations of these two.

Theorem 5.1.9 Let the function $f: E \rightarrow \mathbf{R}$, where $E$ has the Baire property, have positive symmetric derivative everywhere on the set $E$. Then there is an open set $G$ so that $E \backslash G$ is meager and $f$ is increasing on each component of $G$.

Proof. The proof is the repetition of the proof of Theorem 2.2.7. We only need extra condition that the intermediate points $x_{i}, i=1,2,3,4$ may be chosen to form a monotonic sequence (see the comment after Theorem 2.3.6).

Let

$$
V=\left\{[x-t, x+t]: x \pm t \in E \Rightarrow \frac{f(x+t)-f(x-t)}{2 t}>0\right\}
$$

be a symmetric cover relation on $E$. This relation satisfies assumption of Theorem 2.3.6. So for almost every $x \in E$ there is a neighborhood $U_{x}$ so that $x+t \in$ $U_{x} \Rightarrow[x, x+t] \in V^{5}$ and intermediate endpoints are in $E$. Say that we choose $t>0$. 
There are points $x_{0}=x<x_{1}<\cdots<x_{5}=x+t$ such that $x_{i} \in E$ for $i=1,2,3,4$ and $\left[x_{i}, x_{i+1}\right] \in V$ for $i=0, \ldots, 4$. Therefore $f(x+t)-f(x)>0$ so $f$ is increasing at almost every point of $E$.

Theorem 5.1.10 Let the function $f: E \rightarrow \mathbf{R}$, where $E$ is measurable, have positive symmetric derivative everywhere on the set $E$. Then $f$ is locally increasing at almost every point of $E$.

Proof. The proof of this theorem is identical to that for Theorem 5.1.9.

\subsection{Measurability of symmetric derivatives}

The ordinary derivative (of functions defined on $\mathbf{R}$ ) is always a Baire 1 function. So is the case with symmetric derivative for functions from $\mathbf{R}$ to $\mathbf{R}$.

Theorem 5.2.1 (Larson [T2, thm. 7.18]) Let the function $f: \mathbf{R} \rightarrow \mathbf{R}$ be everywhere symmetrically differentiable (allowing infinite values). Then the symmetric derivative of $f$ is in the first Baire class.

For symmetric domains not being the whole $\mathbf{R}$ we have Fact 5.1.8, but note that in this fact the function $f$ is Baire 1 relatively to $\mathbf{Q}$. We do not know whether symmetric derivative must be of any Borel or measurable class when defined on any other domain.

Question 5.2.2 Let $f: A \rightarrow \mathbf{R}$ be a symmetrically differentiable function and let $A$ be a symmetric set. Is the symmetric derivative of $f$ a Borel or measurable function? Does it depend on the size of $A$ ? 
Example 2.2.11, which served as a counterexample in many cases, fails this time. Even though the function $f$ defined there is nonmeasurable, it is symmetrically differentiable and its symmetric derivative is constant equal zero everywhere on the domain.

When the domain is measurable (we drop the assumption of symmetry), then its symmetric derivative is measurable. This follows from a theorem of Preiss and Thomson ([T2, thm. 7.50]) with only minor changes to the proof. In fact, much more can be said about the symmetric derivative in this case or when the domain has the Baire property.

Theorem 5.2.3 Let $f: A \rightarrow \mathbf{R}$ be arbitrary function and let $S D f$ and $D f$ stand for the sets of symmetric differentiability and ordinary differentiability respectively. Then the set $S D f \backslash D f$ contains no measurable set of positive measure and no second category set with the Baire property.

Proof. The proof is similar to the proof of Theorems 5.1.10 and 5.1.9.

We show that in almost every point of $\operatorname{SD} f$ the ordinary derivative of $f$ is not greater that the symmetric derivative at this point. Identical argument can be used to show the converse inequality.

Let $g(x)$ be the symmetric derivative of $f$ and let $h(x)$ be the upper limit of difference quotients. The set $\{x: g(x)<h(x)\}$ can be written as a countable union

$$
\bigcup_{r \in \mathbf{Q}}\{x: g(x)<r<h(x)\} .
$$

By the way of contradiction assume that one of the sets in the union contains a measurable subset of positive measure or second category subset with the Baire 
property. In particular there exists a number $K$ with the property that the set $X=\{x: g(x)<K\} \backslash\{x: h(x) \leq K\}$ is either measurable of positive measure or second category with the Baire property.

We define a symetric cover of $X$ by

$$
V=\{[x-t, x+t]: t>0 \& x \pm t \in A \Rightarrow f(x+t)-f(x-t)<2 K t\} .
$$

Then $V$ satisfies conditons of Theorems 2.3.5 or 2.3.6. So just like in the proof of Theorem 5.1.9 we get that almost every point $x$ of $X$ satisfies for $t>0$ and appropriately small

$$
x \pm t \in A \Rightarrow(f(x+t)-f(x)<K t \& f(x)-f(x-t)<K t) .
$$

Thus at almost every point of $X$ in the sense of measure and category we have $h(x) \leq K$, which contradicts our assumption of the set $X$ being big.

As a corollary we get that if $f: A \rightarrow \mathbf{R}$ is symmetrically differentiable and $A$ is measurable (has the Baire property) then $f$ is almost everywhere (on a residual set) differentiable also in the ordinary sense. So we obtain a corollary which is a counterpart to Theorem 5.2.1. We state the category theorem only as it is similar to Theorem 5.2.1 (the measure couterpart requires different method to be proven).

Theorem 5.2.4 Let $f: E \rightarrow \mathbf{R}$, where $E$ has the Baire property, be symmetrically differentiable (allowing infinite values). Then its symmetric derivative has the Baire property, and there is a meager set $T \subset E$ such that the symmetric derivative restricted to $E \backslash T$ is of Baire class 1.

We remark that Theorem 5.2.4 can be improved to the whole domain as Fact 5.1.7 states that on a set of measure zero and first category symmetric derivative may be any function. 


\section{Bibliography}

[Be] C. L. Belna, Symmetric continuity of real functions, Proc. Amer. Math. Soc. 87 (1983), 99-102.

[Br] A. M. Bruckner, Differentiation of Real Functions, CMR Series vol. 5, Amer. Math. Soc., 1994.

[Ch] M. Chlebík, There are $2^{\mathbf{c}}$ symmetrically continuous functions, Proc. Amer. Math. Soc. 113 (1991), 683-688.

[Ci1] K. Ciesielski, On range of uniformly antisymmetric functions, Real Anal. Exchange 19 (1993-94), 616-619.

[Ci2] K. Ciesielski, Set Theory for the Working Mathematician, London Math. Soc. Student Texts 39, Cambridge Univ. Press 1997.

[Ci3] K. Ciesielski, Set Theoretic Real Analysis, J. Appl. Anal. 3(2) (1997), 143190.

[Ci4] K. Ciesielski, Decomposing symmetrically continuous and SierpińskiZygmund functions into continuous functions, Proc. Amer. Math. Soc. 123 (1999), 3615-3622. 
[CL] K. Ciesielski, L. Larson, Uniformly antisymmetric functions, Real Anal. Exchange 19(1) (1993-94), 226-235.

[CSh] K. Ciesielski, S. Shelah, Uniformly antisymmetric function with bounded range, Real Anal. Exchange 24(2) (1998-99), 615-619.

[CSz] K. Ciesielski, M. Szyszkowski, A symmetrically continuous function which is not countably continuous, Real Anal. Exchange 22 (1996-97), 428-432.

[Fr] H. Fried, Über die symmetrische Stetigkeit von Funktionen, Fund. Math. 29 (1937), 134-137.

[JS] J. Jaskuła, B. Szkopińska, On the set of points of symmetric continuity, An. Univ. Bucureşti Mat. 37 (1988), 29-35.

[Ku] M. Kuczma, An Introduction to the Theory of Functional Equations and Inequalities, Polish Scientific Publishers PWN, Warsaw 1985.

[K] K. Kuratowski, Topology, Vol. I, Acad. Press, New York, N.Y., 1966.

[L] L. Larson, Symmetrical analysis: a survey, Real Anal. Exchange 9 (1983-84), $154-178$.

[N] A. Nowik, private communication.

[Pe] I. N. Pesin, On the measurability of symmetrically continuous functions, Teor. Funktsiǔ Funktsional. Anal. i Prilozhen 5 (1967), 99-101.

[Pr] D. Preiss, A note on symmetrically continuous functions, Časopis Pěst. Mat. 96 (1971), 262-264. 
[SZ] E. M. Stein, A. Zygmund, On the differentiability of functions, Studia Math. 23 (1960), 295-307.

[Sz1] M. Szyszkowski, Points of weak symmetric continuity, Real Anal. Exchange 24 (1998-99), 807-813.

[Sz2] M. Szyszkowski, Symmetrically continuous functions on proper subsets of the real line, Real Anal. Exchange, to appear.

[T1] B. S. Thomson, Real Functions, Springer-Verlag, Lecture Notes in Math. 1170, 1985.

[T2] B. S. Thomson, Symmetric properties of real functions, Marcel Dekker, 1994.

[Uh] J. Uher, Symmetric continuity implies continuity, Trans. Amer. Math. Soc. 293 (1986), 421-429. 